\title{
Stimuli-responsive polymeric micelles for drug delivery and cancer therapy
}

This article was published in the following Dove Press journal:

International Journal of Nanomedicine

\author{
Qing Zhoul,* \\ Li Zhang $2, *$ \\ TieHong Yang' \\ Hong Wu' \\ 'Department of Pharmaceutical \\ Analysis, School of Pharmacy, Air \\ Force Military Medical University, \\ Xi'an, People's Republic of China; \\ ${ }^{2}$ State Key Laboratory of Military \\ Stomatology, Air Force Military \\ Medical University, Xi'an, People's \\ Republic of China \\ *These authors contributed equally \\ to this work
}

\begin{abstract}
Polymeric micelles (PMs) have been widely investigated as nanocarriers for drug delivery and cancer treatments due to their excellent physicochemical properties, drug loading and release capacities, facile preparation methods, biocompatibility, and tumor targetability. They can be easily engineered with various functional moieties to further improve their performance in terms of bioavailability, circulation time, tumor specificity, and anticancer activity. The stimuli-sensitive PMs capable of responding to various extra- and intracellular biological stimuli (eg, acidic pH, altered redox potential, and upregulated enzyme), as well as external artificial stimuli (eg, magnetic field, light, temperature, and ultrasound), are considered as "smart" nanocarriers for delivery of anticancer drugs and/or imaging agents for various therapeutic and diagnostic applications. In this article, the recent advances in the development of stimuliresponsive PMs for drug delivery, imaging, and cancer therapy are reviewed. The article covers the generalities of stimuli-responsive PMs with a focus on their major delivery strategies and newly emerging technologies/nanomaterials, discusses their drawbacks and limitations, and provides their future perspectives.
\end{abstract}

Keywords: nanomedicine, polymeric micelles, stimuli-responsive, drug delivery, cancer therapy

\section{Introduction}

Cancer remains one of the leading causes of human death worldwide. Although significant progress has been made in cancer biology, genomics, proteomics, and clinical oncology during the past several decades, anticancer treatments are unsatisfactory and the overall survival of many cancer patients stays low. ${ }^{1}$ Chemotherapy is one of the most commonly used cancer treatment options in the clinic. Typically, chemotherapy drugs are toxic chemicals and administrated systemically via either parenteral or oral routes. However, these drugs often have drawbacks, including poor solubility and bioavailability, unfavorable pharmacokinetics, and nonselective biodistribution, which may complicate their clinical use and lead to undesirable side effects., ${ }^{2,3}$

The nanotechnology-based medicines, that is, nanomedicines, have shown excellence in drug delivery, diagnosis, and therapy, particularly in cancer research. Nanoscale drug delivery systems (NDDSs) or nanocarriers play an important role in nanomedicine to overcome the aforementioned drawbacks of chemotherapy drugs. Compared to traditional drug delivery systems, nanocarriers have shown greater potential in improving drug bioavailability, prolonging drug circulation time, controlling drug release, and targeting tumor. In addition to performing these basic functions, the newly emerging technology and nanomaterials may offer extra functions or opportunities to nanomedicines/nanocarriers for various purposes of cancer diagnosis and treatments. ${ }^{4,5}$ Various nanocarriers have been developed, of which the most commonly studied are the
Correspondence: Hong Wu

Department of Pharmaceutical Analysis, School of Pharmacy, Air Force Military Medical University, 169 West Changle Street, Xi'an 7I0032, People's

Republic of China

Tel +862984776823

Email wuhong@fmmu.edu.cn
International Journal of Nanomedicine 20 I 8: I 3 292 |-2942

2921

Dovepress ff in 0

http://dx.doi.org/10.2147/IJN.S158696 (c) (i) (3) 2018 Zhou et al. This work is published and licensed by Dove Medical Press Limited. The full terms of this license are available at https://www.dovepress.com/terms.php cc) and incorporate the Creative Commons Attribution - Non Commercial (unported, v3.0) License (http://creativecommons.org/licenses/by-nd/3.0/). By accessing the work you hereby accept the Terms. Non-commercial uses of the work are permitted without any further permission from Dove Medical Press Limited, provided the work is properly attributed. For permission for commercial use of this work, please see paragraphs 4.2 and 5 of our Terms (https://www.dovepress.com/terms.php). 
organic nanocarriers, such as liposomes, polymeric micelles (PMs), and dendrimers, and inorganic nanocarriers, such as carbon nanotubes, silica nanoparticles, gold nanoparticles, magnetic nanoparticles, and quantum dots. ${ }^{6,7}$

PMs are one of the first polymeric self-assemblies. Over the past few decades, they have received tremendous attention in biomedical sciences, especially as drug carriers, since they provide several physicochemical and biological advantages over other types of nanoassemblies, including easy preparation, simple architecture, drug solubilization, improved biocompatibility, pharmacokinetics, and biodistribution, and additional opportunities for further engineering. ${ }^{8,9}$ It is worth noting that several drug-loaded PM formulations have been approved for clinical trials for cancer treatments, and some PMs showed the promising anticancer activity (Table 1). ${ }^{10,11}$ For instance, Genexol ${ }^{\circledR}$-PM is a paclitaxel (PTX)-loaded PM formulation which consists of a block copolymer, poly(ethylene glycol) (PEG)-poly(D,L-lactide) (PLA). PTX is one of the most commonly used chemotherapy drugs for treating ovarian, breast, lung, cervical, and pancreatic cancers. However, it has very low water solubility and is currently used as a solution in Cremophor EL/ethanol $(1: 1, \mathrm{w} / \mathrm{w})$. Unfortunately, the Cremophor may cause unwanted side effects such as hypersensitivity, nephrotoxicity, and neurotoxicity. Moreover, the dose-limiting toxicity such as neuropathy and neutropenia is another concern. In preclinical

Table I Current clinical trials of anticancer drug-loaded polymer micelles

\begin{tabular}{|c|c|c|c|c|c|}
\hline Name & $\begin{array}{l}\text { Block } \\
\text { copolymer }\end{array}$ & Drug & Indications & $\begin{array}{l}\text { Clinical } \\
\text { status }\end{array}$ & References \\
\hline \multirow[t]{16}{*}{ Genexol ${ }^{\circledR}$-PM } & PEG-PLA & PTX & Recurrent breast cancer & Phase IV & NCT009I 2639 \\
\hline & & & Breast cancer & Phase III & NCT00876486 \\
\hline & & & Ovarian cancer & Phase II & NCT00877253, \\
\hline & & & & & NCT008867I7, \\
\hline & & & & & NCT0I 276548 \\
\hline & & & Non-small-cell lung cancer & Phase II & NCT0I023347, \\
\hline & & & & & NCT0I770795 \\
\hline & & & Bladder and ureter cancer & Phase II & NCT0I426I26 \\
\hline & & & Pancreatic cancer & Phase II & NCT00III 904 \\
\hline & & & Metastatic breast cancer & Phase II & NCT0I784I20 \\
\hline & & & Recurrent/metastatic adenocarcinoma of the pancreas & Phase II & NCT02739633 \\
\hline & & & Hepatocellular carcinoma & Phase II & NCT030085I2 \\
\hline & & & Locally advanced head and neck squamous cell carcinoma & Phase II & NCT0I689194 \\
\hline & & & Gynecologic cancer & Phase I & NCT02739529 \\
\hline & & & Ovarian cancer & Phase I & NCT00877253 \\
\hline & & & Pancreatic cancer & Phase I & NCT00882973 \\
\hline NKI05 & PEG-PAPB & PTX & Breast cancer & Phase III & NCT0I 644890 \\
\hline \multirow[t]{5}{*}{ NC-6004 } & PEG-PGlu & DDP & Head and neck neoplasms & Phase I & NCT028I7II3 \\
\hline & & & Locally advanced and metastatic pancreatic cancer & Phase I/II & NCT009I074I \\
\hline & & & Squamous cell carcinoma of the head and neck & Phase I/II & NCT03109I58 \\
\hline & & & Solid tumors & Phase I/II & NCT02240238 \\
\hline & & & Pancreatic cancer & Phase III & NCT02043288 \\
\hline \multirow[t]{5}{*}{ NKOI 2} & PEG-PGlu & SN-38 & Refractory solid tumors & Phase I & NCT00542958 \\
\hline & & & Metastatic colorectal cancer & Phase I & NCT0I 238939 \\
\hline & & & Metastatic triple-negative breast cancer & Phase I & NCT0I238952 \\
\hline & & & Small-cell lung cancer & Phase II & NCT00951613 \\
\hline & & & Triple-negative breast cancer & Phase II & NCT0095I054 \\
\hline SPI049C & PEG-PPO-PEG & DOX & Advanced adenocarcinoma & Phase III & $118-120$ \\
\hline \multirow[t]{4}{*}{ BIND-0I4 } & PEG-PLA & Docetaxel & Advanced or metastatic cancer & Phase I & NCT0I300533 \\
\hline & & & KRAS-positive or squamous cell non-small-cell lung cancer & Phase II & NCT02283320 \\
\hline & & & Non-small-cell lung cancer & Phase II & NCT0I792479 \\
\hline & & & Metastatic castration-resistant prostate cancer & Phase II & NCT0I8I 2746 \\
\hline NC-4016 & PEG-PGlu & OXA & Advanced solid tumor or lymphoma & Phase I & NCT03। 68035 \\
\hline \multirow[t]{2}{*}{ Nanoxel ${ }^{\circledR} \mathrm{M}$} & PEG-PLA & Docetaxel & Head and neck squamous cell carcinoma & Phase II & NCT02639858 \\
\hline & & & Non-muscle-invasive bladder cancer & Phase III & NCT02982395 \\
\hline NK9II & PEG-PAPB & DOX & Various solid tumors & Phase II & 121,122 \\
\hline
\end{tabular}

Abbreviations: PM, polymeric micelle; PEG, poly(ethylene glycol); PLA, poly(D,L-lactide); PTX, paclitaxel; PAPB, polyaspartate modified with 4-phenyl-butanol; PGlu, poly(glutamic acid); DDP, cisplatin; SN-38, 7-ethyl-I0-hydroxy-camptothecin; PPO, poly(propylene oxide); DOX, doxorubicin; OXA, oxaliplatin. 
studies, Genexol-PM demonstrated a threefold increase in the maximum tolerated dose and a significantly increased antitumor efficacy compared with free PTX. Currently, a number of clinical studies over Genexol-PM are underway.

In order to exert anticancer effects, drugs have to be released from the PMs efficiently and specifically in tumor tissues or inside tumor cells. However, highly stable PMs may result in slow and incomplete drug release and lower the efficacy of the loaded drugs. Therefore, various stimuliresponsive PMs have been developed for "smartly" controlling drug release as well as drug efficacy. ${ }^{12,13}$ The idea of stimuli-responsive drug delivery systems comes from the fact that the tumor tissue possesses a variety of unique features compared with the normal tissue, including acidic $\mathrm{pH}$, altered redox potential, and overexpressed proteins and enzymes. In addition, stimuli such as temperature, light, ultrasound, and magnetic fields can be applied to the tumor site externally to allow PMs to bypass the biological barriers, reach their targets, and release their loaded drugs. This article aims to summarize and discuss the recent advances of stimuliresponsive PMs in drug delivery and cancer therapy.

\section{Polymeric micelles}

PMs are spherical colloidal particles with a size typically ranging from 10 to $100 \mathrm{~nm}$. They are composed of amphiphilic copolymers that have distinct hydrophobic and hydrophilic blocks. ${ }^{14}$ Usually, these copolymers are amphiphilic di-block (hydrophilic-hydrophobic) or tri-block (hydrophilichydrophobic-hydrophilic) polymers. The most commonly used hydrophilic blocks are PEG with a molecular weight of 2-15 kDa, while the hydrophobic blocks typically are polyesters, polyethers, or polyamino acids, such as poly(L-aspartic acid), PLA, poly( $\varepsilon$-caprolactone) (PCL), and poly(propylene oxide) (PPO). ${ }^{15}$ The defining characteristic of PMs is the polymers' ability of spontaneous self-assembly into nanoscale aggregates. In aqueous solutions, the hydrophobic blocks selfassociate into a semisolid core surrounded by the hydrophilic segments as a corona/shell. The hydrophilic shell provides steric stability and minimizes nonspecific uptake by the reticuloendothelial system (RES), resulting in the prolonged circulation time in the body. ${ }^{16,17}$ Given the fact that many anticancer drugs are water insoluble, the hydrophobic core of PMs provides a perfect place for drug loading. By this way, the insoluble drugs can be "solubilized". The critical micelle concentration (CMC) is the minimum concentration of copolymers required for micelle formation. At the concentration lower than $\mathrm{CMC}$, the copolymers exist as monomers throughout the solution, while at the concentration around or above $\mathrm{CMC}$, the polymer chains associate and the self-assemblies/micelles are formed. Most reported PMs showed quite low CMC in comparison with low-molecular-weight surfactant micelles. ${ }^{17}$

In the early 1980s, Gros et al first reported PMs as drug delivery vehicles for cancer treatment. ${ }^{18}$ Since then, a great number of PMs have been developed to deliver various anticancer drugs and other bioactive molecules for cancer diagnosis and therapy. As drug carriers, the coreshell-structured PMs offer several advantages and unique properties for cancer-targeted drug delivery. First of all, as mentioned previously, PMs can load the water-insoluble drugs by physical entrapment or chemical conjugation, resulting in greatly increased drug solubility. As an example, 7-ethyl-10-hydroxy-camptothecin (SN-38), a highly effective anticancer agent, which acts as a DNA topoisomerase I inhibitor, cannot be administered intravenously due to its poor water solubility and high toxicity. By conjugation of the drug to PEG-poly(glutamic acid) (NK012), the solubility of the drug is significantly increased. As a result, the IC50 value of NK012 is deceased up to 5.8 times compared to that of free SN-38. ${ }^{19}$ In solid tumors, the tumor specificity of the nanodrug carriers considerably relies on the enhanced permeability and retention (EPR) effect, a passive targeting strategy..$^{20,21}$ For passive targeting, nanoparticles have to circulate in the blood for a long period of time to ensure that sufficient drugs are delivered to the tumor tissue, in which their size plays a very important role. Nanoparticles smaller than $10 \mathrm{~nm}$ are easily eliminated through the renal glomeruli, whereas nanoparticles larger than $100 \mathrm{~nm}$ can be cleared from the bloodstream rapidly by the liver and spleen. ${ }^{22-24}$ On the basis of this knowledge, PMs with a suitable size (10-100 $\mathrm{nm}$ ) can avoid their quick clearance from the body, thus allowing for their accumulation in the tumor tissues via the EPR effect. In addition, the biocompatible polymeric corona of PMs can suppress nonspecific interactions with biological components and lead to reduced recognition by the RES, prolonging the circulation time in the bloodstream. Nevertheless, the EPR effect of PMs may not always benefit the treatments particularly in the human clinical trials due to the heterogeneity and complexity of the tumor environment. Thus, PMs can be further modified with ligands for active targeting to increase their tumor selectivity and intracellular drug delivery but reduce their systemic toxicity and other adverse effects. ${ }^{25}$ Compared to nontargeted PMs, a number of studies have showed the superior results of the active-targeting PMs, such as improved cellular uptake, cytotoxicity, and tumor regression, making the active targeting strategy an important addition to the 
passive targeting PMs. All these advantages demonstrate PMs may be a promising and powerful drug platform for cancer therapeutic applications.

\section{Stimuli-responsive PMs}

Despite the successful applications of PMs as anticancer drug carriers, some problems remain. For example, PMs are highly associated with premature drug release in the circulation and inadequate drug release upon accumulation in the targeted tissue. ${ }^{26}$ Ideally, the drug is retained in the PMs during circulation but is released after accumulation in the tumor interstitium. The premature drug release can be prevented by appropriate micelle stabilization or enhancement of drug interaction in the micelle core via specific bonding strengths like hydrogen bonds or cleavable covalent bonds. As to achieving tumor-targeted drug delivery and adequate release, a very effective way is the design of stimuli-responsive PMs. With the increasing knowledge of tumor biology, we know that tumor tissues possess a low $\mathrm{pH}$ in solid tumors with an even lower $\mathrm{pH}$ inside tumor cells, overexpressed specific enzymes, high levels of glutathione (GSH) in the cytoplasm, as well as a higher temperature compared with the normal tissues. These unique features can be utilized as internal triggers along with external stimuli such as magnetic field, ultrasound, and light to permit the destabilization of stimuli-responsive PMs and achieve temporally and spatially controlled drug delivery and release. ${ }^{13}$ The strategies for the development of stimuli-responsive PMs used in cancer therapy are reviewed individually below (typical stimuliresponsive PMs are summarized in Table 2).

\section{$\mathrm{pH}$-responsive $\mathrm{PMs}$}

The $\mathrm{pH}$-responsive PMs have recently emerged as important nanocarriers for anticancer drug/imaging agent delivery. ${ }^{27-30}$ As a result of enhanced metabolic rates and increased aerobic glycolysis, the microenvironment of most solid tumors is intrinsically acidic ( $\mathrm{pH}$ 6.5-7.2), while the $\mathrm{pH}$ value in the blood and normal tissue is about 7.4. Moreover, an even lower acidic $\mathrm{pH}$ is found in endosomes $(\mathrm{pH}$ 6.5-5.5) and lysosomes ( $\mathrm{pH} 4.5-5.0) .{ }^{31}$ Accordingly, the difference in $\mathrm{pH}$ has been widely exploited to achieve tumor site- or organellespecific activation of $\mathrm{pH}$-responsive $\mathrm{PMs}$ and on-demand drug release. The $\mathrm{pH}$ sensitivity of polymers mainly results from the protonation of ionizable groups or the degradation of $\mathrm{pH}-$ sensitive linkages. Therefore, one possible approach to impart $\mathrm{pH}$ sensitivity to PMs is to incorporate the ionizable groups into the micellar polymers to ionize at the tumor extracellular or intracellular acidic $\mathrm{pH}$. After the micelle decomposition

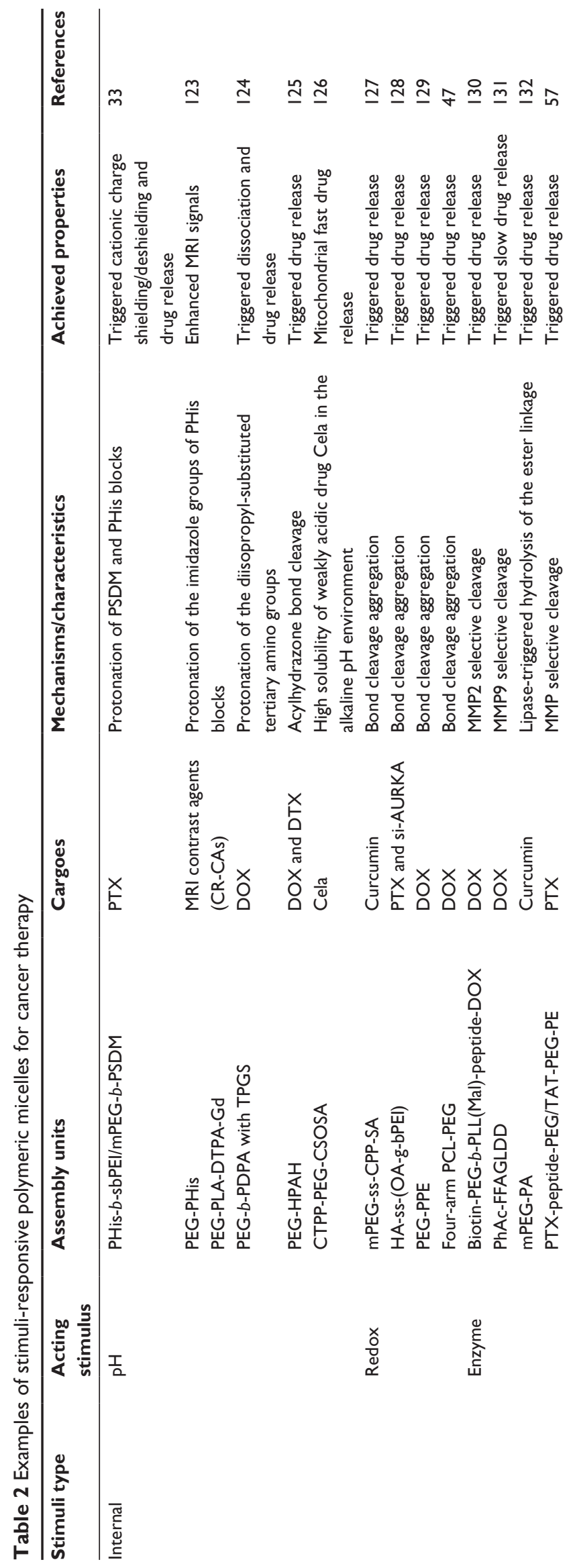




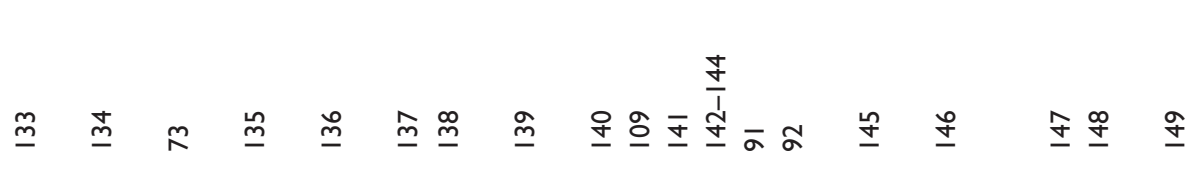

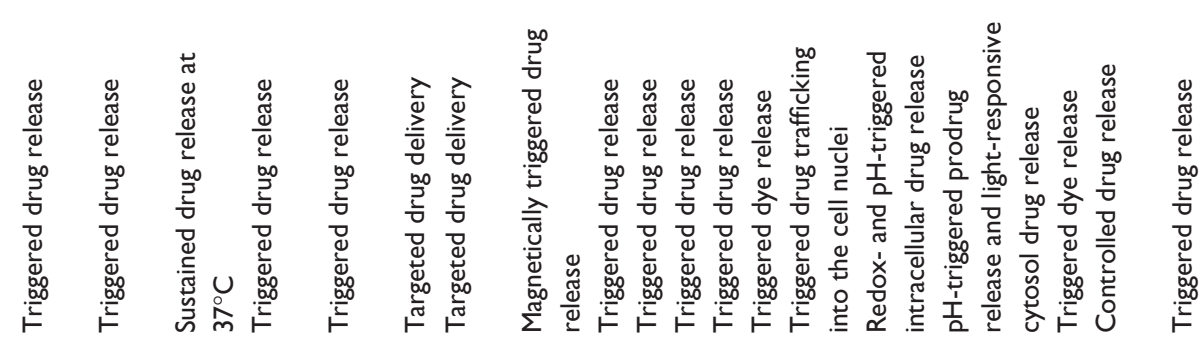
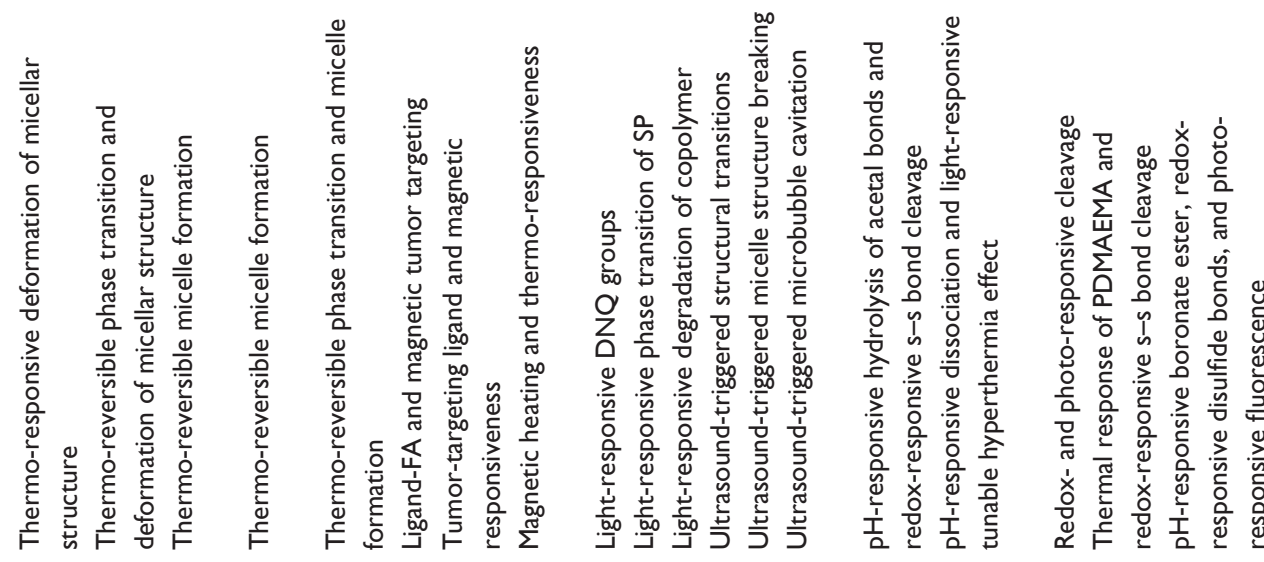

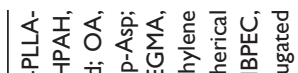

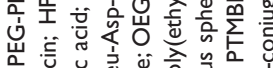

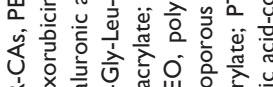

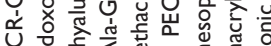

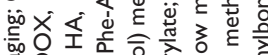
等

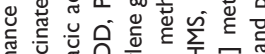

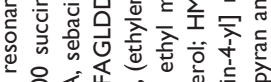

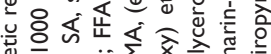

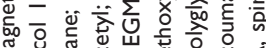

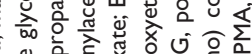

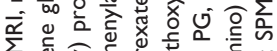

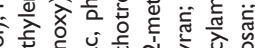

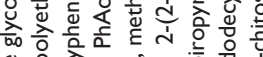

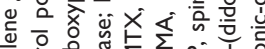

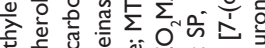

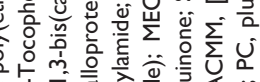

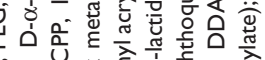

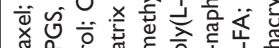

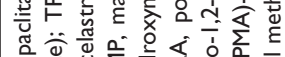

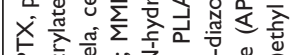

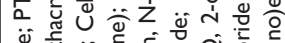

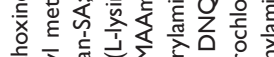

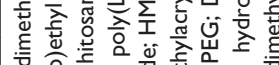

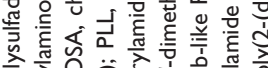

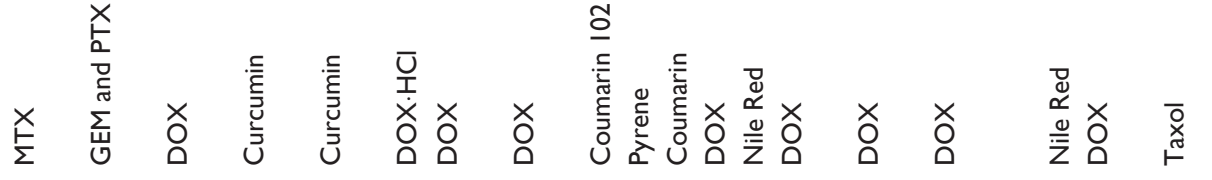

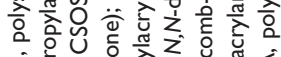

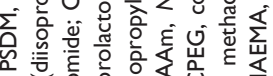
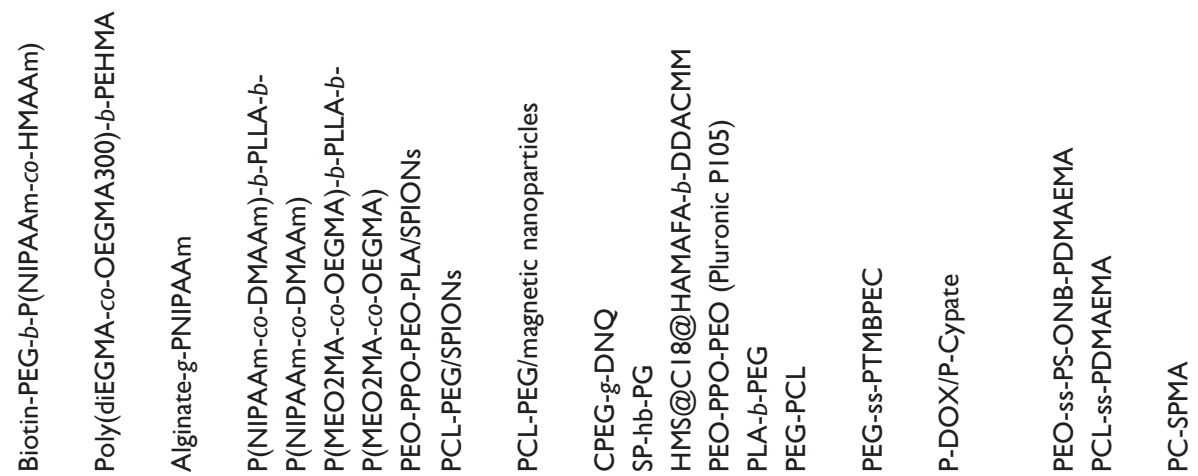

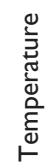

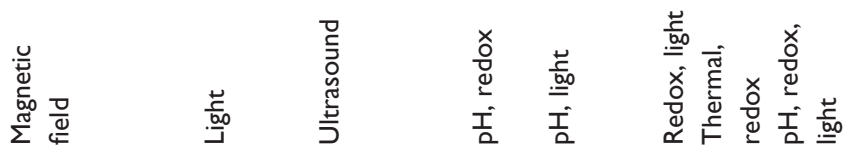

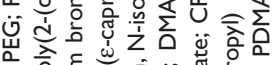

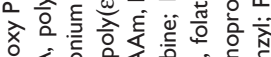

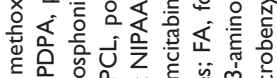
o है

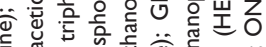

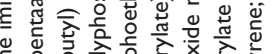

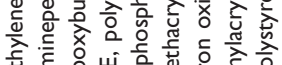

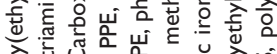

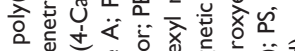

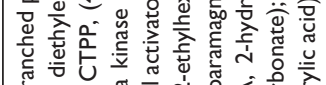

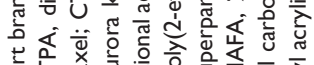

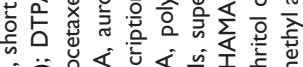

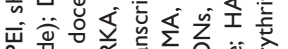

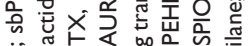

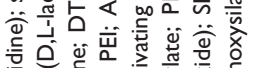

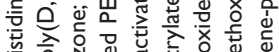

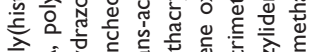

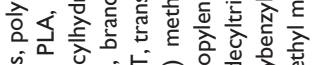

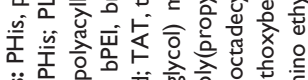

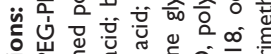

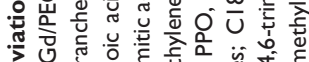

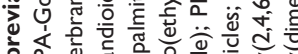


or destabilization, their payloads are released at the tumor tissues. For example, a pH-sensitive mixed micelle was prepared by $\mathrm{Wu}$ et al for cytosolic delivery of doxorubicin (DOX), which was composed of two block polymers, 1,2-distearoyl-sn-glycero-3-phosphorylethanolamine-PEG and $\mathrm{pH}$-responsive poly(histidine) (PHis)-PEG. ${ }^{32}$ PHis is a commonly employed $\mathrm{pH}$-sensitive polymer as the imidazole ring has an electron lone pair on the unsaturated nitrogen that endows PHis with an amphoteric nature by protonationdeprotonation. Such systems could not only stabilize the micelles at neutral and tumor extracellular $\mathrm{pH}\left(\mathrm{pH}_{\mathrm{e}}\right)$ but also facilitate micelle's disintegration and then result in quick drug release in response to the low endocytic $\mathrm{pH}$. Similarly, Hu et al utilized polysulfadimethoxine (PSDM) and PHis to construct a micellar system to realize sequential $\mathrm{pH}$ responsiveness at the tumor extracellular and endosomal $\mathrm{pH} .{ }^{33}$ Cationic micelles were prepared from a block copolymer of PHis-poly(ethylene imine) (PEI), which was shielded by the negatively charged methoxy PEG (mPEG)-PSDM at pH 7.4 and deshielded at the tumor extracellular $\mathrm{pH}$ due to the $\mathrm{pH}$-responsive sulfonamide group of PSDM. After endocytosis, PHis was protonated in the endosomal compartment, leading to dissociation of the micelle and destabilization of the endosomal membrane to promote the delivery of drugs to the cytosol. The in vivo results demonstrated that the PMs showed significant anticancer efficacy and deep penetration into tumor tissues.

Another common $\mathrm{pH}$-sensitive strategy utilizes the degradation of $\mathrm{pH}$-sensitive linkages at compartments with lower $\mathrm{pH}$. In recent years, one major branch of our research focused on stimuli-responsive nanodrug delivery systems, and our group has made several attempts to construct $\mathrm{pH}-$ sensitive PMs for cancer therapy. ${ }^{32,34,35}$ In a study, a novel delivery system based on luteinizing hormone-releasing hormone (LHRH)-PEG-PHis-DOX/DOX-trans-activating transcriptional activator (TAT) acid-sensitive micelle was developed, as shown in Figure 1, which could dissociate and release DOX-TAT when responding to the tumor extracellular $\mathrm{pH} .{ }^{34}$ Such systems could efficiently go across the cell membrane and reach the cytosol of the multidrug-resistant cancer cells, resulting in the remarkable antitumor efficacy and negligible systematic toxicity. Zhou et al developed an $N$-(2-hydroxypropyl)methacrylamide (HPMA) copolymerbased micellar drug delivery system, in which the hydrophobic drug DOX and $\beta$-sitosterol were conjugated to the hydrophilic HPMA polymer backbone via a $\mathrm{pH}$-sensitive hydrazone linker. ${ }^{36}$ The $\mathrm{pH}$-responsive linker was stable at physiological $\mathrm{pH}$ but could be easily hydrolyzed at acidic $\mathrm{pH}$. In the in vitro studies, the PMs could quickly collapse with $80 \%$ of the drug released at $\mathrm{pH} 5$, the lyso/endosomal $\mathrm{pH}$.
In the in vivo studies, the PMs showed significantly higher anticancer effect.

As we know, the positively charged carriers could effectively interact with the negatively charged cell membrane by electrostatic attraction and trigger efficient cell internalization. ${ }^{37,38}$ However, for in vivo drug delivery, positively charged surface is not favorable due to nonspecific interactions and resultant short circulation half-life. . $^{39,40}$ Thus, strategies that make use of charge conversion or charge reversal are highly required and always in pursue for $\mathrm{pH}$ sensitive drug delivery, in which PMs are usually neutral or negatively charged at physiological $\mathrm{pH}$ and are capable of converting their surface charge into positive one in response to the tumor extracellular $\mathrm{pH}$. In our previous work, a dual $\mathrm{pH}$-sensitive charge-reversing poly( $\beta$-L-malic acid) (PMLA)based micellar nanocomplex (PMLA-PEI-DOX-TAT@PEG2,3-dimethylmaleic anhydride (DMMA)) was developed for effective tumor-targeted drug delivery, enhanced cellular uptake, and intracellular drug release, which is illustrated in Figure 2. ${ }^{35}$ The prepared micellar nanocomplex showed a negative surface charge at the physiological $\mathrm{pH}$, while at the tumor extracellular environment, charge reversal of PEG-DMMA took place and the positively charged and TATexposed PMs were released, resulting in enhanced cellular internalization. Moreover, after endocytosis, the PMs showed $\mathrm{pH}$-sensitive quick drug release at the endo/lysosomal $\mathrm{pH}$ because of the $\mathrm{pH}$-sensitive linker.

\section{Redox-responsive PMs}

Redox potential has been regarded as a viable biomarker to distinguish between the extracellular and intracellular environments, as well as between the tumor and normal tissues due to the difference in the GSH concentration. The concentration of GSH in cytoplasm (approximately $2-10 \mathrm{mM}$ ) could be 100-1,000 times higher than that in the extracellular environments (approximately $2-10 \mu \mathrm{M}$ ). ${ }^{41-43}$ In comparison with healthy tissues, some tumor tissues are found to be highly reducing and hypoxic, with the intracellular GSH concentration being at least four times higher than that of the normal cells. ${ }^{41,43}$ In the past decade, a tremendous progress was made in the development of redox-responsive nanovehicles for targeted intracellular drug or gene delivery as the redox response is exceedingly fast and efficient in the presence of high concentration of GSH in the tumor or intracellular environments. In addition, unlike $\mathrm{pH}$-sensitive nanocarriers that are usually designed to release drugs in the lyso/endosomal compartments, redox-responsive systems can disassemble and release drugs in the cytosol and cell nucleus. Disulfide bonds, prone to rapid cleavage by 


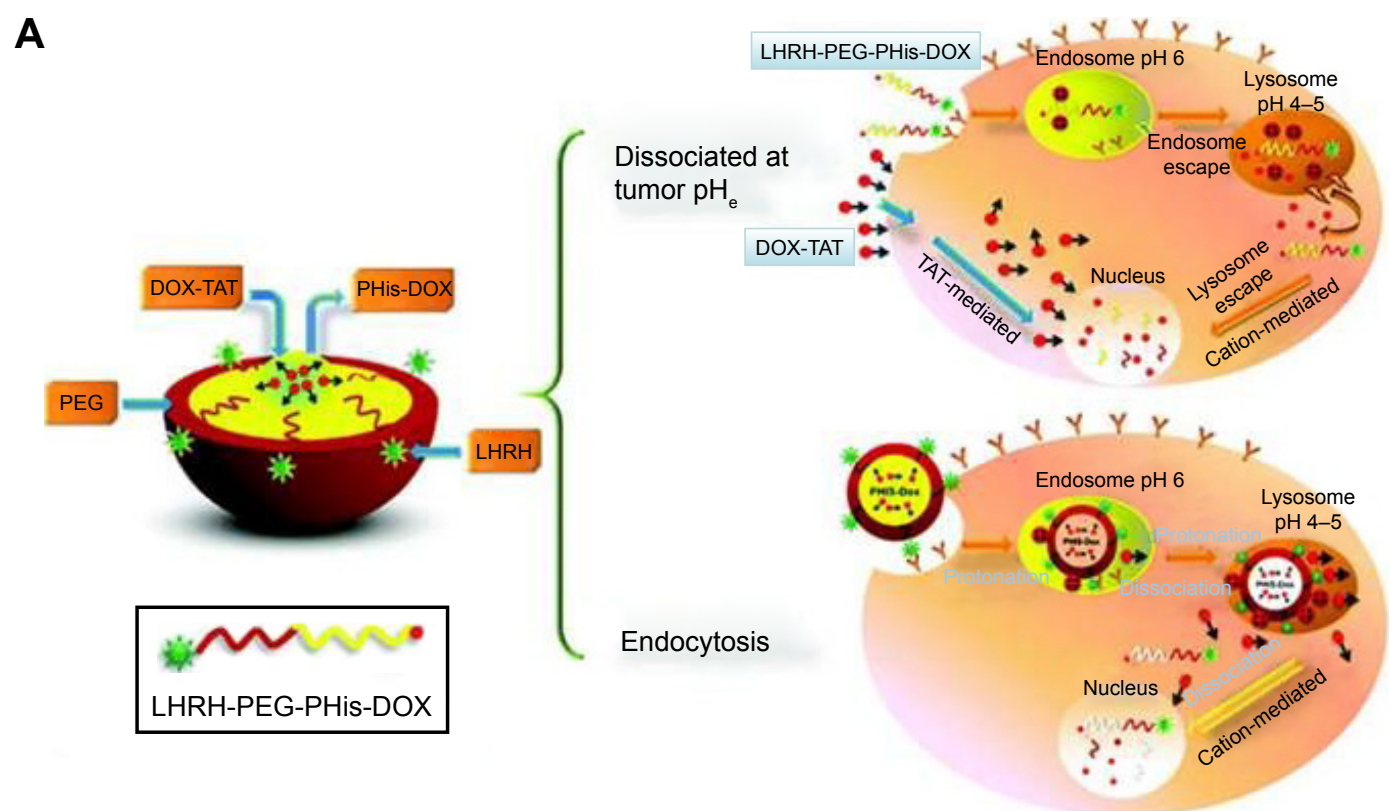

$\mathbf{B}$
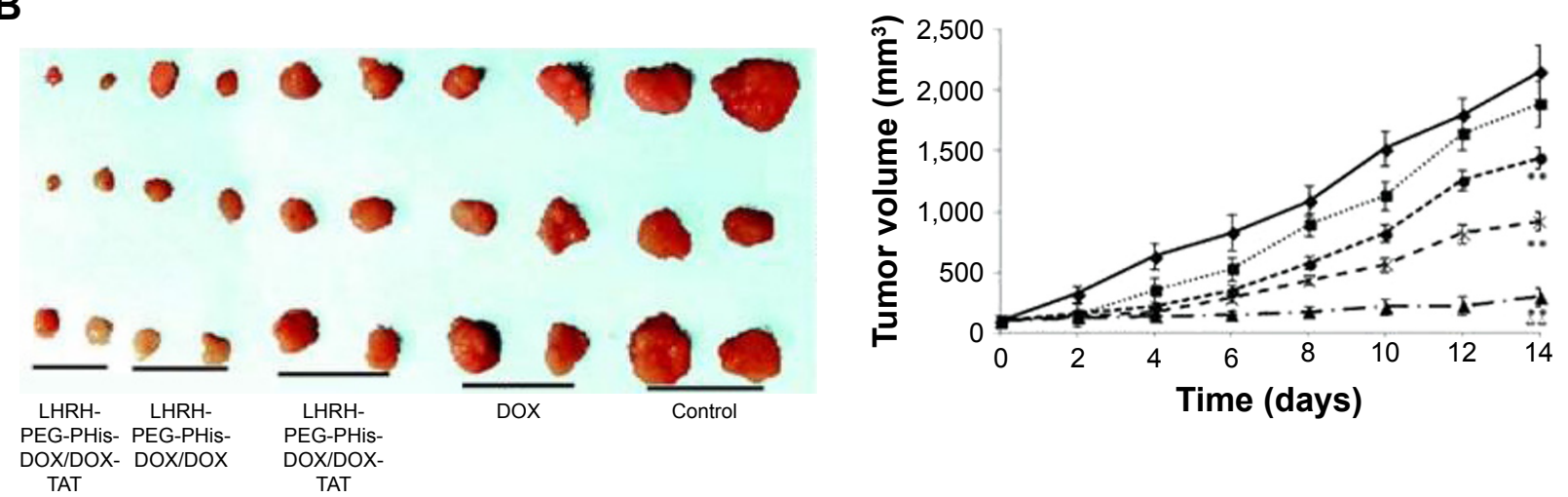

Figure I (A) Delivery strategy of LHRH-PEG-PHis-DOX/DOX-TAT micelles. (B) Images of the tumors on day 14 after the inoculation of A2780/DOXR tumor cells in the mice and in vivo antitumor efficacy.

Notes: Reprinted from Yang T, Li F, Zhang H, et al. Multifunctional pH-sensitive micelles for tumor-specific uptake and cellular delivery. Polym Chem. 20I 5;6(8): I373-1382. ${ }^{34}$ With permission from the Royal Society of Chemistry. Copyright 2014 Royal Society of Chemistry.

Abbreviations: LHRH, luteinizing hormone-releasing hormone; PEG, poly(ethylene glycol); PHis, poly(histidine); DOX, doxorubicin; TAT, trans-activating transcriptional activator.

GSH, are commonly used to gain redox sensitivity. The chemical structure of disulfide-containing cross-linking agents is shown in Figure 3. Until now, a number of redoxresponsive PMs have been designed by incorporating a disulfide bond either in the hydrophobic blocks or between the hydrophobic and hydrophilic segments. ${ }^{44}-46$ Under the redox environment, these PMs can be reductively degradable or rapidly disassembled to release payloads.

Shi et al used four-arm PCL-PEG copolymers to develop a multifunctional star-shaped micellar system by combination of active targeting ability and redox-responsive behavior. ${ }^{47}$ The redox-responsive behavior was realized by connecting PCL and PEG via disulfide bonds, and active targeting ability was achieved by the introduction of folate (FA) ligands to the end groups of the hydrophilic segment, as shown in Figure 4. DOX was trapped into the micelles during the self-assembly of the star-shaped PCL-PEG copolymers. The prepared redox-responsive PMs could be specifically internalized by tumor cells through the FA receptor-mediated endocytosis, and the disulfide bonds could be immediately cleaved in response to the intracellular high level of GSH, resulting in quick DOX release. The same group reported another redoxresponsive polyanhydride-based micelle for cancer therapy. ${ }^{48}$ The amphiphilic polyanhydride copolymer contained disulfide bonds between the hydrophilic and hydrophobic segments. Redox sensitivity of the micelles was demonstrated by the changes of the micellar size, morphology, and molecular weight. Particularly, an approximate zero-order in vitro drug (curcumin) release mode in a short time was observed in a reducing and acidic environment which was similar to that of the tumor cell, leading to significant in vitro and in vivo antitumor activities. 
A
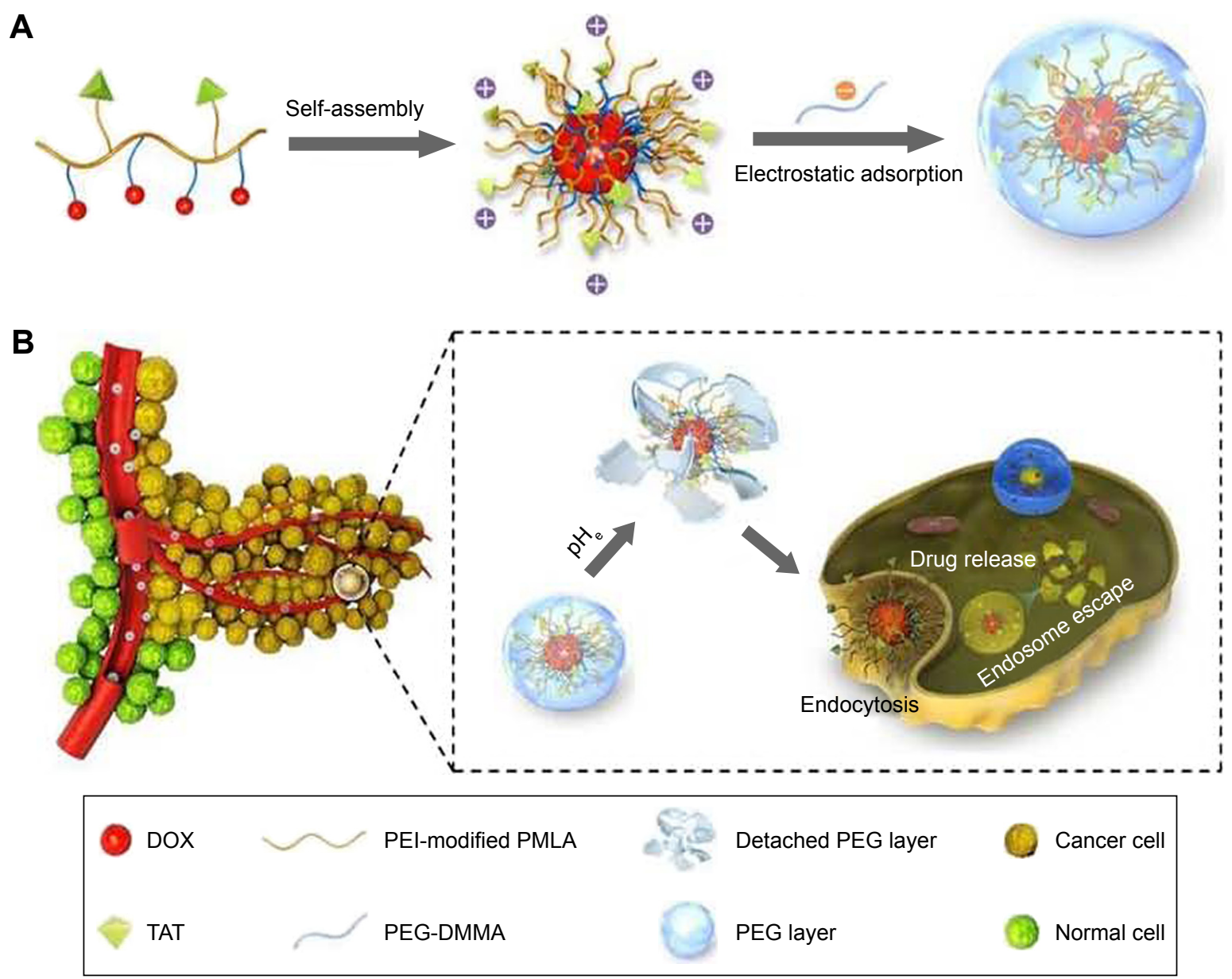

Figure 2 (A) Preparation of nanocomplex PMLA-PEI-DOX-TAT@PEG-DMMA. (B) Schematic illustration of the dual pH-sensitive DOX-loading nanocomplex with the charge-conversional function for effective tumor-targeted drug delivery, enhanced cellular uptake, and intracellular drug release.

Notes: Reprinted from Zhou Q, Hou Y, Zhang L, et al. Dual-pH sensitive charge-reversal nanocomplex for tumor-targeted drug delivery with enhanced anticancer activity. Theranostics. 2017;7(7):1806-1819.35 Creative Commons Attribution (CC BY-NC) license, available from: https://creativecommons.org/licenses/by-nc/4.0/.

Abbreviations: PMLA, poly( $\beta$-L-malic acid); PEI, poly(ethylene imine); DOX, doxorubicin; TAT, trans-activating transcriptional activator; PEG, poly(ethylene glycol); DMMA, 2,3-dimethylmaleic anhydride.

\section{Enzyme-responsive PMs}

Enzymes play critical roles in many biological and metabolic processes inside the body due to their exceptional specificity and outstanding catalytic properties. ${ }^{49}$ The dysregulation of enzyme expression and activity has been observed in a variety of different pathological situations and is associated with many diseases. For example, the expression of several types of enzymes like proteases, peptidases, and lipases in solid tumors is often greater compared to their concentrations in normal tissues since high concentrations of these enzymes play a very important role in tumor cell growth, angiogenesis, invasion, and metastasis. ${ }^{50-52}$ Dysregulated enzymes have been considered as biomarkers for diagnostics and prognostics in different types and stages of cancer, which are also emerging as promising biological triggers for targeted cancer therapy. Using enzymes as a trigger could provide a number of benefits as most enzymes catalyze chemical reactions under mild conditions (eg, low temperature and neutral $\mathrm{pH}$ value), however, many conventional nonenzymatic chemical reactions may not happen under such conditions. Moreover, enzymes possess highly exceptional selectivity for their substrates, thus allowing for specific, complicated, biologically inspired chemical reactions. Based on these principles, in the past few years, a wide variety of NDDSs have been developed as the enzyme-responsive systems for selective and efficient targeted drug delivery, including polymeric nanoparticles, liposomes, and inorganic nanoparticles. ${ }^{49,53-56}$ The enzyme responsiveness can be rendered to the PMs for anticancer drug delivery by incorporating specific moieties in their main chain or side groups that can be selectively recognized and degraded by overexpressed enzymes in the extra/intracellular environment of the tumor, enabling tumor-targeted and spatiotemporal drug release with reduced unwanted side effects in normal tissues.

Matrix metalloproteinases (MMPs), a kind of extracellular matrix (ECM)-remodeling proteases, have been found 
<smiles>O=C(O)CCSSCCC(=O)O</smiles><smiles>O=C(CCSSCCC(=O)ON1C(=O)CCC1=O)ON1C(=O)CCC1=O</smiles>
Dithiobis (succinimidylpropionate)<smiles>C=CC(=O)NCCSSCCNC(=O)C=C</smiles>

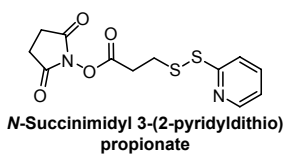<smiles>O=C(CCSSc1ccccn1)NCCCCCC(=O)ON1C(=O)CCC1=O</smiles>

LC-SPDP

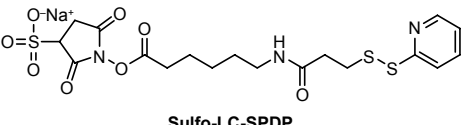

$\mathrm{H}_{2} \mathrm{~N} \sim \mathrm{S}^{-} \mathrm{S} \sim \mathrm{NH}_{2}$

Cystamine

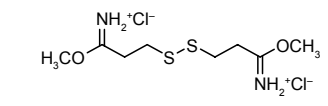

Dimethyl-3,3'-dithiobispropionimidate.2HCl<smiles>C=C(C)C(=O)OCCSSCCOC(=O)C(=C)C</smiles>

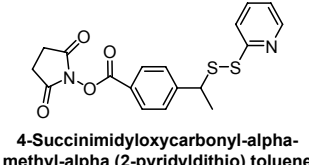
methyl-alpha (2-pyridyldithio) toluen
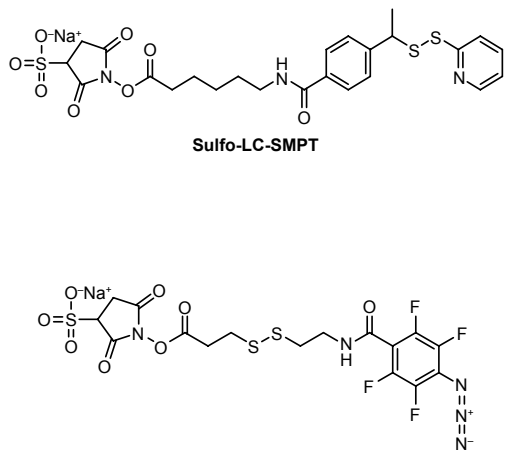

Sulfo-SFAD
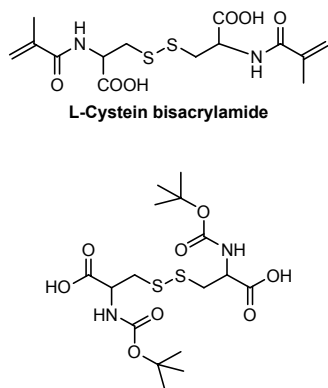

$N, N$-Bis-(terbutoxycarbonyl) cysteine<smiles>NCCSSc1ccccn1</smiles>

2-(Pyridyldithio)-ethylamine<smiles>Cc1c(CC(=O)NCCSSCCC(=O)ON2C(=O)C3C(=O)N(O)C(=O)C32)c(=O)oc2cc(N=N)ccc12</smiles>

Sulfo-SAED<smiles>N=[N+]=Nc1ccc([N+](=O)[O-])c(C(=O)NCCSSCCC(=O)ON2C(=O)CC(S(=O)(=O)O)C(=O)C2O)c1</smiles>

Sulfo-SAND

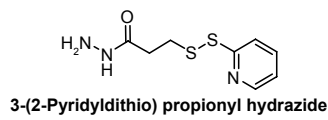

Figure 3 Chemical structures of disulfide-containing redox-sensitive linkers.

Abbreviations: SAND, sulfosuccinimidyl 2-(m-azido-o-nitrobenzamido)-ethyl-1,3'-dithio propionate; SAED, sulfosuccinimidyl-2-(7-amino-4-methylcoumarin-3-acetamido)ethyl-I,3-dithiopropionate; LC-SPDP, succinimidyl 6-(3(2-pyridyldithio)propionamido)hexanoate; LC-SMPT, 4-succinimidyloxycarbonyl- $\alpha$-methyl- $\alpha$ (2-pyridyldithio)toluene; SFAD, sulfosuccinimidyl-(perfluoroazidobenzamido)-ethyl-I,3'-dithiopropionate.

highly associated with tumor invasion and metastasis. ${ }^{50,51}$ They are a family of zinc- and calcium-dependent endopeptidases that are responsible for the degradation and remodeling of the ECM and are relatively overexpressed in almost all tumors compared with normal tissues. Thus, MMPs can be employed to achieve enzyme-responsive site-specific drug release. Among them, MMP2 and MMP9 are the most widely explored enzymes for enzymatic drug delivery. For example, a unique tumor-targeted micellar drug delivery platform was reported by Zhu et al, which was prepared by self-assembly of the block copolymers of MMP2-sensitive $\mathrm{PEG}_{2000}-\mathrm{PTX}$ conjugate, transactivating transcriptional activator peptide$\mathrm{PEG}_{1000}$-phosphoethanolamine (PE), and $\mathrm{PEG}_{1000}-\mathrm{PE}$, acting as MMP2-sensitive functional polymer, cell-penetrating enhancer, and nanocarrier building block, respectively
(Figure 5). ${ }^{57}$ The MMP2 sensitivity of $\mathrm{PEG}_{2000}$-peptide-PTX as well as the whole micelle formation was well characterized. Compared to nonsensitive counterparts, like free PTX and conventional micelles, both in vitro and in vivo studies indicated that this novel design showed superior cell internalization, cytotoxicity, tumor targeting, and antitumor efficacy, which had great potential for effective intracellular delivery of drug into cancer cells. More recently, they designed another MMP2-sensitive multifunctional PM for tumorspecific co-delivery of siRNA and hydrophobic drugs. ${ }^{58}$ This micellar platform was constructed by an MMP2-sensitive copolymer (PEG-pp-PEI-PE) via self-assembly, which exhibited excellent stability, efficient siRNA condensation by PEI, PTX solubilization in the lipid core, and tumor targeting via both the EPR effect and MMP2 sensitivity. 


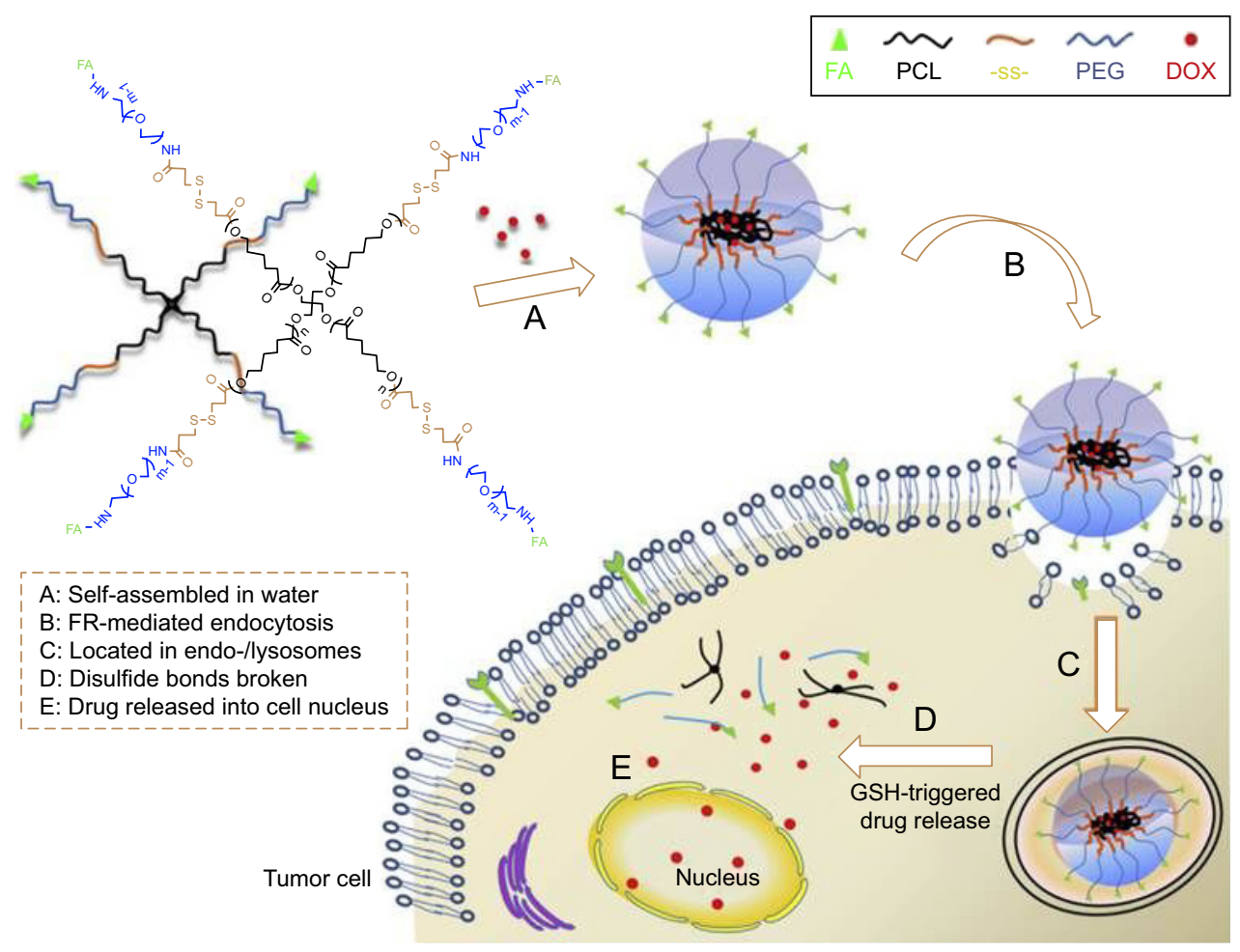

Figure 4 Schematic illustration of DOX-loaded star-shaped micelles based on FA-functionalized and redox-responsive star copolymer (star-PCL-ss-PEG-FA) for FRmediated endocytosis and GSH-triggered intracellular drug release.

Notes: Reprinted from Shi C, Guo X, Qu Q, Tang Z, Wang Y, Zhou S. Actively targeted delivery of anticancer drug to tumor cells by redox-responsive star-shaped micelles. Biomaterials. 20I4;35(30):87II-8722. ${ }^{47}$ With permission from Elsevier. Copyright 2014 Elsevier.

Abbreviations: DOX, doxorubicin; FA, folate; PCL, poly( $\varepsilon$-caprolactone); FR, folate receptor; GSH, glutathione; PEG, poly(ethylene glycol).

\section{Thermo-responsive PMs}

Temperature, which acts as either an internal or an external stimulus, is one of the most often investigated stimuli for drug delivery. Alteration of the temperature in specific tissues can either occur due to certain diseases (eg, tumor, inflammation, or infection) or be achieved by the application of an external heating source/device..$^{59,60}$ In addition, the elevated temperature, or "hyperthermia", can also inflict additional damage to tumor cells, such as inhibition of DNA synthesis and repair, disruption of microtubules, and alteration of the receptor expression. Therefore, temperature/thermo-responsive drug delivery systems are among the most investigated stimuli-responsive strategies for cancer therapy. ${ }^{61-64}$ The thermo-sensitive PMs comprise polymers with thermo-responsive blocks which undergo a sharp change of their physical properties in response to the change of temperature to destabilize the micelles and trigger drug release. Thermo-responsive polymers tend to undergo a phase transition at a certain temperature, known as lower critical solution temperature (LCST). ${ }^{65-67}$ Below the LCST, the polymers are soluble in water by forming hydrogen bonds between water and the polymer chains, but become insoluble upon heating (above the LCST) due to disruption of hydrogen bonds. Moreover, the polymers can be modulated by introduction of hydrophobic or hydrophilic comonomers to obtain LCST values within a desired range $\left(37^{\circ} \mathrm{C}-42^{\circ} \mathrm{C}\right)$, higher than normal body temperature but lower than the hyperthermia of tumors.

The most frequently used thermo-responsive polymer for the preparation of thermo-sensitive PMs is $\operatorname{poly}(N$ isopropylacrylamide) (PNIPAAm) with an LCST of around $32^{\circ} \mathrm{C}$, which is close to the physiological temperature of the human body ${ }^{68-71}$ The LCST of PNIPAAm can be optimized by random copolymerization with other monomers in order to get improved targeting and drug release. For example, Panja et al developed a smart thermo-responsive micelle from novel four-arm star-shaped block copolymers, namely PE-PCL- $b$ PNIPAAm-FA and PE-PCL- $b$-poly( $N$-vinylcaprolactam)-FA (Figure 6). ${ }^{72} \mathrm{~A}$ series of temperature-responsive polymers with specific LCST (ranging from $30^{\circ} \mathrm{C}$ to $39^{\circ} \mathrm{C}$ ) were synthesized by tailoring the block length of the thermoresponsive segments. A high rate of DOX release (78.57\% after 24 hours) at a temperature above LCST can be achieved due to the temperature-induced size reduction (57\%) of the 
A

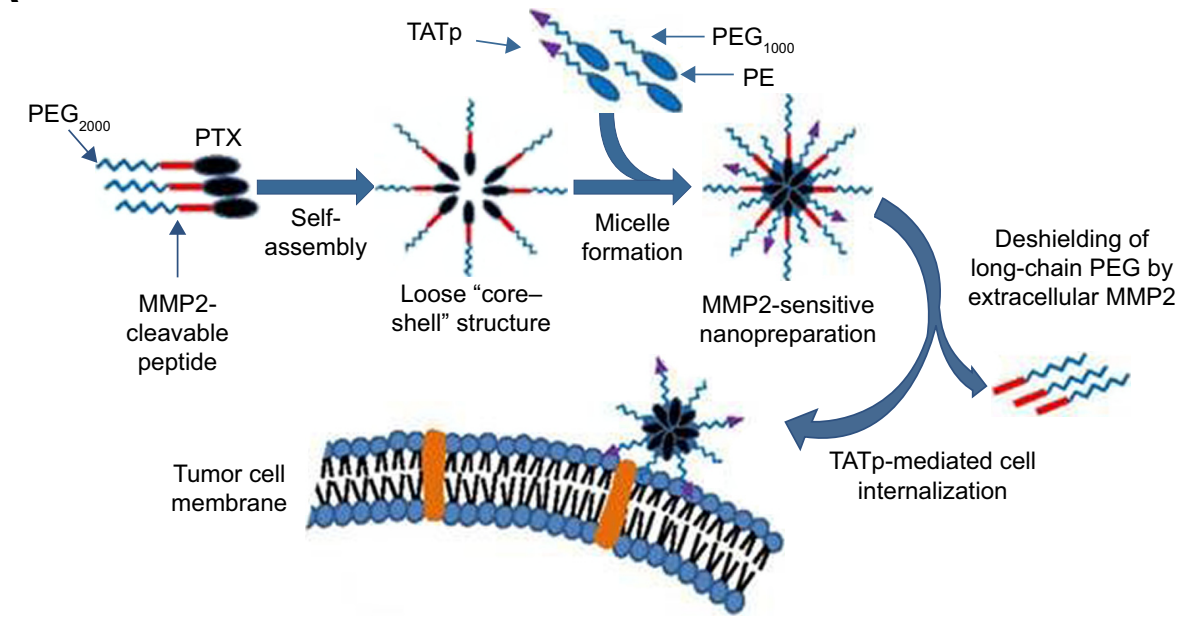

B

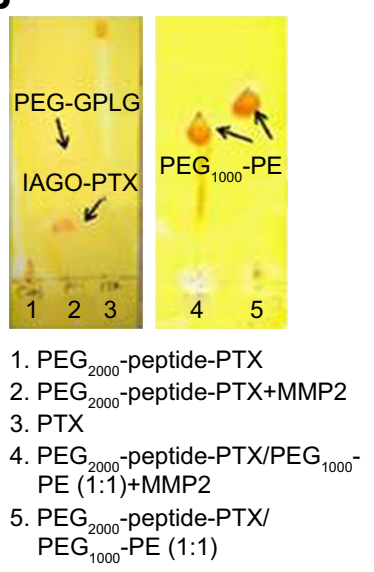

C
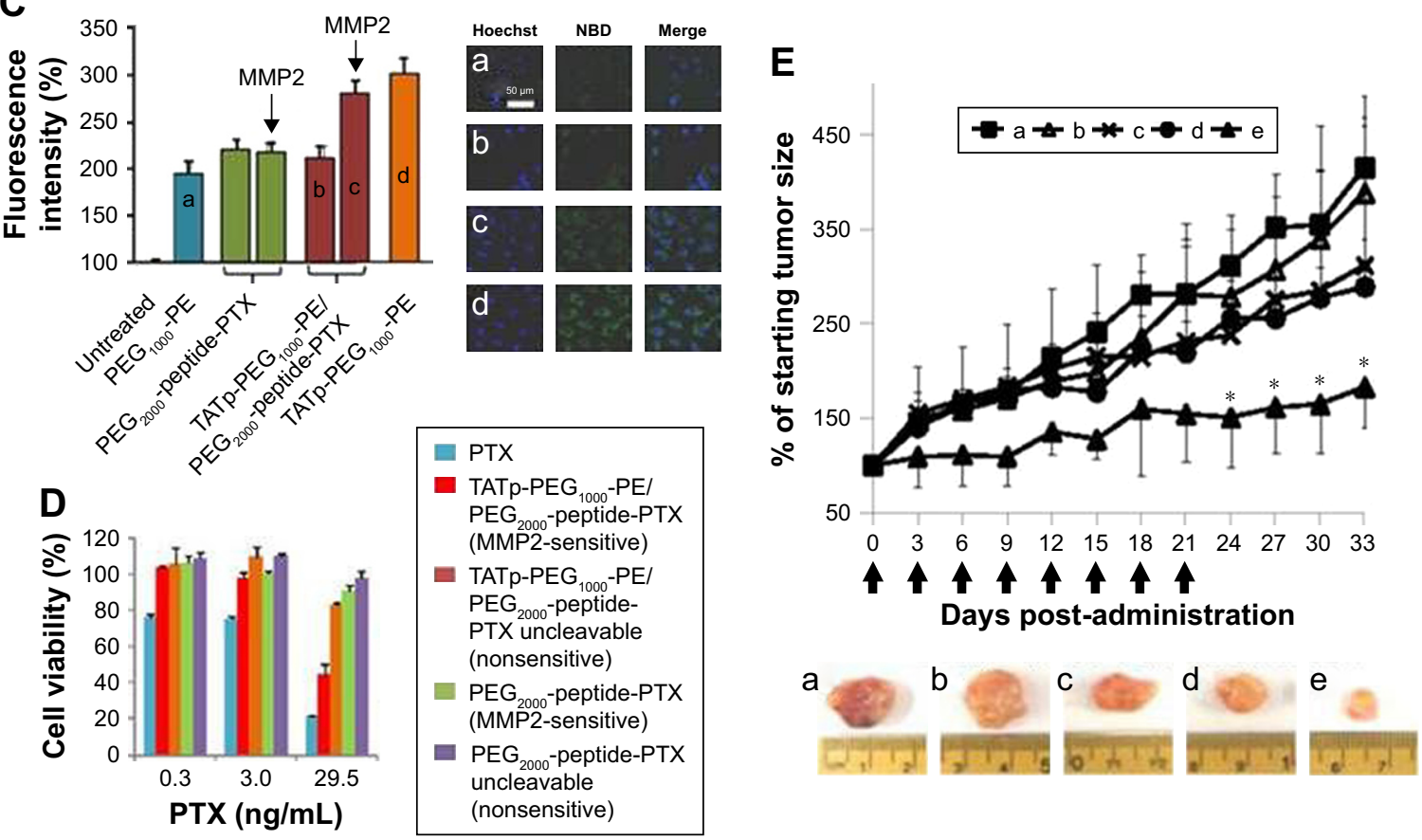

Figure 5 (A) Drug delivery strategy. (B) Enzymatic cleavage. To determine the digestion of PEG $_{2000}$-Peptide-PTX (left) and its nanopreparation (right), the samples were treated with $5 \mathrm{ng} / \mu \mathrm{L}$ MMP2 followed by TLC and Dragendorffs reagent staining. (C) Cellular uptake in A549 cell monolayer. Cells were treated with NBD-PE-labeled formulations (green) for 2 hours before measurement. (a) PEGI000-PE, (b) TATP-PEGI000-PE, (c and d) TATP-PEGI000-PE/PEG2000-peptide-PTX. For FACS (left), cells were trypsinized and collected. For confocal microscopy (right), cells were fixed and stained with Hoechst 33342. (D) Cytotoxicity of the nanopreparations in A549 cell monolayer. Cells were treated with moderate-to-low doses of PTX formulations for 72 hours before CellTiter-Blue Cell Viability Assay. (E) Tumor growth inhibition (\% of the starting tumor volume). (a) HBSS, (b) TATp-PEG 1000-PE/PEG2000-peptide-PTX uncleavable, (c) PEG2000-PE/PTX, (d) Free PTX, (e) TATp-PEG 1000-PE/PEG2000peptide-PTX. $* P<0.05$ compared with other groups.

Notes: Reprinted from Zhu L, Wang T, Perche F, Taigind A, Torchilin VP. Enhanced anticancer activity of nanopreparation containing an MMP2-sensitive PEG-drug conjugate and cell-penetrating moiety. Proc Natl Acad Sci USA. 20 I 3; I 10(42): 17047-17052.57 With permission from the National Academy of Sciences. Copyright 201 3 National Academy of Sciences.

Abbreviations: PEG, poly(ethylene glycol); PTX, paclitaxel; MMP2, matrix metalloproteinase 2; TLC, thin-layer chromatography; NBD, Nitro-2-I,3-BenzoxaDiazol-4-yl; PE, phosphoethanolamine; FACS, fluorescence-activated cell sorting; TATp, transactivating transcriptional activator peptide; GPLG, Gly-Pro-Leu-Gly; IAGQ, Ile-Ala-Gly-Gln.

PMs. Moreover, the DOX release rate can also be tuned by on-demand administration of temperature. The in vivo study showed a drastic inhibition of the tumor volume by $\sim 83.9 \%$ without any significant systemic toxicity after administration of the prepared DOX-loaded PMs into the C6 glioma rat model. Similarly, Ahn et al used amphiphilic alginate$g$-PNIPAAm to prepare a DOX-loaded and near-infrared (NIR) fluorescent dye-tagged PM for cancer imaging and therapy. ${ }^{73}$ Amino-PNIPAAm was conjugated to the alginate backbone by carbodiimide chemistry, the obtained 
A

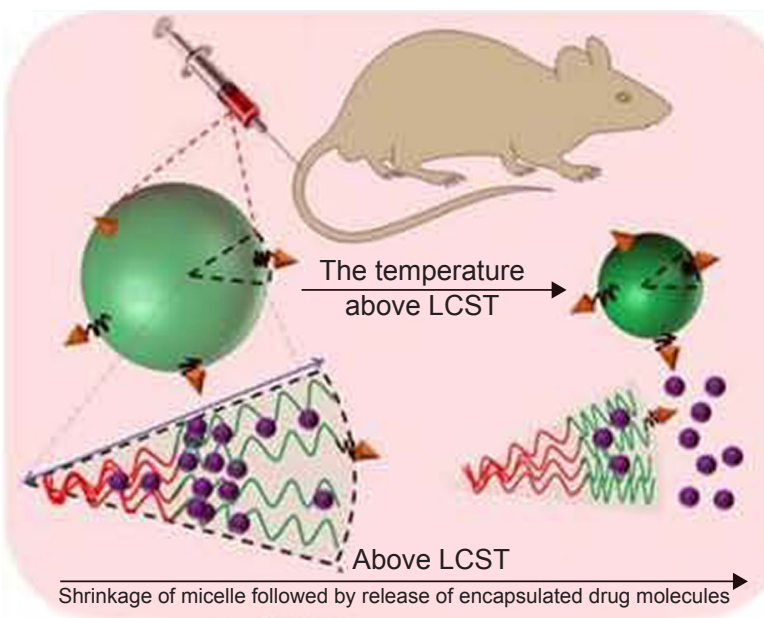

C
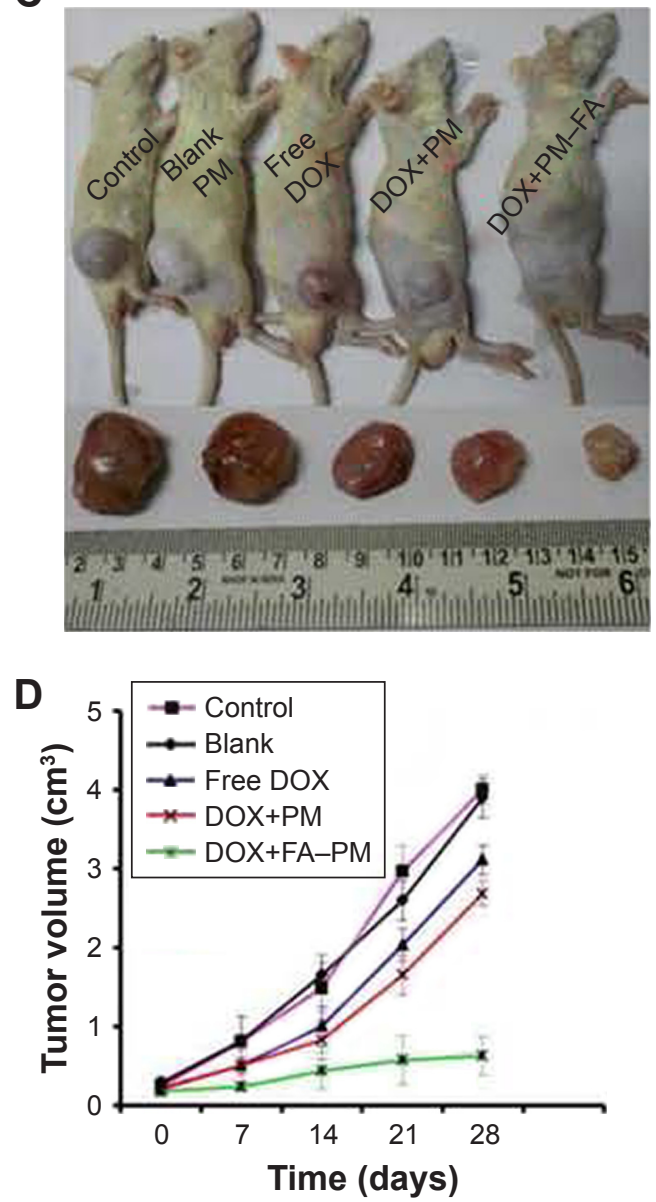

B
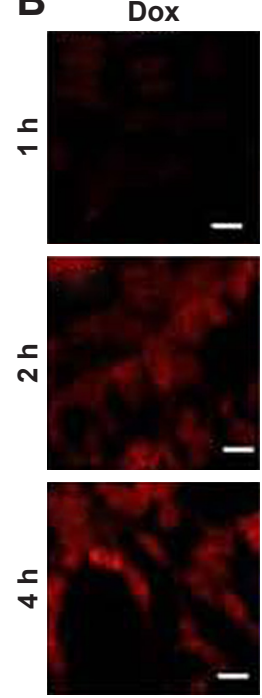

E
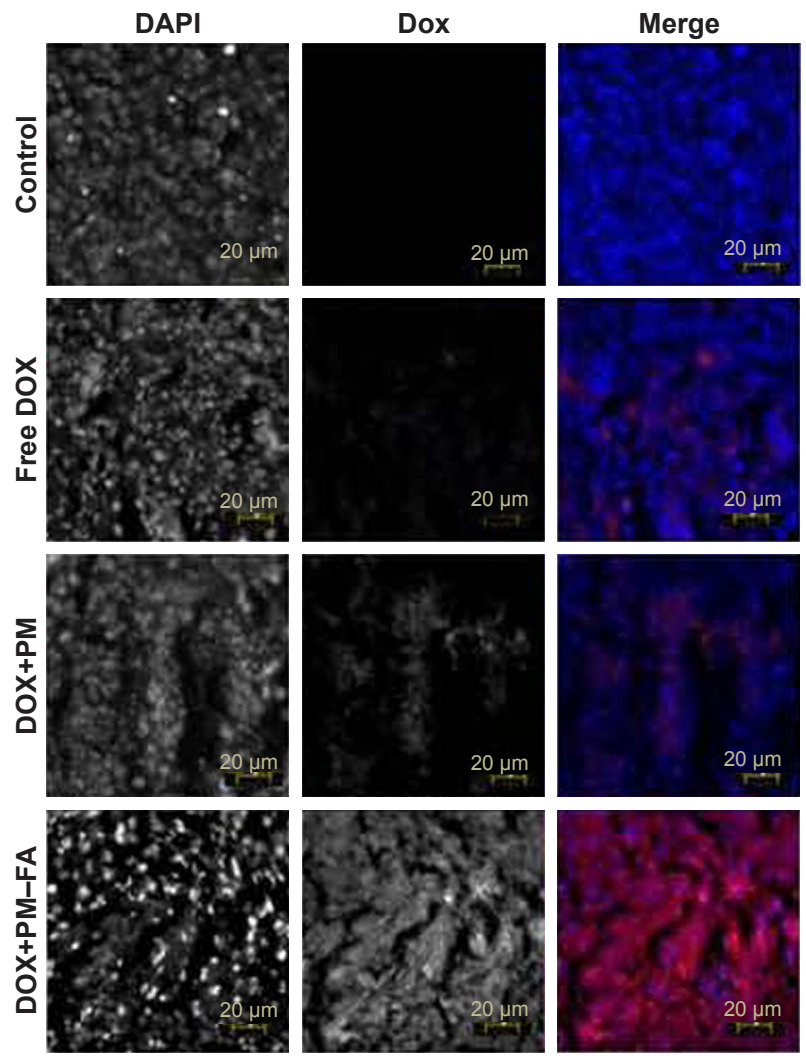

Figure 6 (A) Schematic presentation of temperature-induced shrinkage of micelles followed by drug release. (B) Fluorescence microscopic image of DOX-loaded FA-PM-treated C6 glioma cell line over a time span of I-4 hours. The nuclei were stained with DAPI. (C) Tumor volume of the rat. (D) Variation of tumor volume with time. (E) Microscopic image of in vivo accumulation of control, free DOX, DOX-loaded PMs, and DOX-loaded FA-PMs into the tumor tissue.

Notes: Fluorescence microscopies: red for DOX; blue for DAPI. Reprinted with permission from Panja S, Dey G, Bharti R, et al. Tailor-made temperature-sensitive micelle for targeted and on-demand release of anticancer drugs. ACS Appl Mater Interfaces. 20I6;8(I9):I 2063-I2074. ${ }^{72}$ Copyright 2016 American Chemical Society. Abbreviations: DOX, doxorubicin; FA, folate; PM, polymeric micelle; DAPI, 4',6-diamidino-2-phenylindole; LCST, lower critical solution temperature.

alginate-g-PNIPAAm was dissolved in distilled water at room temperature, and self-assembled PMs were formed at $37^{\circ} \mathrm{C}$. The in vitro result showed a sustained release of DOX from the PMs at $37^{\circ} \mathrm{C}$. The resultant DOX-loaded alginate- $g$-PNIPAAm PMs exhibited excellent anticancer efficacy in a mouse model without any significant side effects, indicating high potential for the application of cancer imaging and therapy. 


\section{Magnetic field-responsive PMs}

Magnetic field-responsive PMs, typically incorporating the therapeutic payloads along with a magnetically active component, could be used not only for tumor targeting under an external magnetic field but also for quick drug release induced by the increased temperature caused by the alternating magnetic field, displaying great potential for cancer treatment. ${ }^{74,75}$ In addition, owning to its deep penetration, magnetic field represents a promising trigger for the in vivo application. Iron oxide nanoparticles with a size less than $10 \mathrm{~nm}$, like magnetite $\left(\mathrm{Fe}_{3} \mathrm{O}_{4}\right)$ and maghemite $\left(\mathrm{Fe}_{2} \mathrm{O}_{3}\right)$, are most commonly used to impart the magnetic sensitivity. ${ }^{76-78}$ They are also referred to as superparamagnetic iron oxide nanoparticles (SPIONs) due to their property of superparamagnetism and small sizes. They can be attracted by a magnetic field, but do not retain the residual magnetism upon removal of the field. Apart from magnetic drug targeting, SPIONs have also been widely used for other biomedical applications including magnetic resonance imaging (MRI), magnetic transfection, and magnetic hyperthermia. ${ }^{79}$ The magnetic hyperthermia is a good example. Under alternating magnetic field, SPIONs would lead to the increased local temperature, which could be utilized to either directly kill tumors or make them more susceptible in combination with chemotherapy. Therefore, magnetic-sensitive polymeric nanoparticles have been explored for remotely controlled drug release as well as a combination of cancer therapy and diagnosis.

Targeted delivery of therapeutics and diagnostics using nanotechnology holds great promise to minimize the side effects of conventional chemotherapy and enable specific and real-time detection of diseases. In this regard, Wei et al recently reported the development of a clickable and imageable nanovehicle assembled from multiblock polyurethanes for precise tumor theranosis..$^{80}$ The soft segments of the polymers were based on detachable PEG and degradable PCL, and the hard segments were constructed from lysine and cystine derivatives bearing reduction-responsive disulfide linkages and click-active alkynyl moieties, allowing for post-conjugation of targeting ligands via a click chemistry (Figure 7). They found that the cleavage of PEG corona bearing a pH-sensitive benzoic-imine linkage could act as an on-off switch, which was capable of activating the clicked targeting ligands under extracellular acidic environment, followed by triggering the core degradation and payload release inside tumor cells. Furthermore, in combination with SPION entrapped in the micellar core, the prepared PMs exhibited excellent MRI contrast effects and T2 relaxation in vitro, as well as magnetically guided MRI and multimodal targeting of therapeutics to tumor precisely, resulting in significant inhibition of cancer with minimal side effect.
In a similar study, FA-bovine serum albumin (BSA)functionalized SPION-loaded PMs were prepared by Li et al for tumor targeting and MRI. ${ }^{81}$ The SPION-loaded PMs were prepared by self-assembly of amphiphilic poly $(2,2,3,4,4,4$ hexafluorobutyl methacrylate-co-methacryloxyethyl trimethyl ammonium chloride)-g-methoxy PEG monomethacrylate (poly(HFMA-co-MOTAC)-g-PEGMA) copolymers and oleic acid-modified $\mathrm{Fe}_{3} \mathrm{O}_{4}$ nanoparticles, and then functionalized with FA-BSA by electrostatic interaction. In vivo MRI conducted on the nude mice bearing the Bel-7402 xenografts after bolus intravenous administration showed excellent tumor targeting and MRI capabilities, especially at 24 hours post-injection. A multifunctional theranostic micellar drug delivery system utilizing cationic poly(2-(dimethylamino)ethyl methacrylate) (PDMA)- $b$-PCL micelles was developed for the delivery of various agents, including $\mathrm{SN}-38$, ultrasmall SPIONs, and siRNA that targets human vascular endothelial growth factor (VEGF) ${ }^{82}$ The prepared PMs could passively target tumor regions and synergistically facilitate VEGF silencing and chemotherapy, as well as acted as a negative MRI contrast agent in T2-weighted imaging, indicating a powerful tool for the cancer diagnosis and tracking of the therapeutic outcomes.

\section{Ultrasound-responsive PMs}

Due to the ease of administration, low cost, and deep penetration, ultrasound has been widely used in diagnostic and therapeutic applications for many years, and has been proven to be an efficient noninvasive approach for the diagnosis and treatment of a wide range of health problems such as osteoporosis and cardiovascular diseases. ${ }^{83}$ Additionally, ultrasound has been extensively explored as a trigger for spatiotemporally controlled release of active agents encapsulated in nanopreparations at the desired site, thus reducing unwanted side effects. In the past years, a number of NDDSs with ultrasound-triggered release, including nanocapsules, ${ }^{84-86}$ nanoemulsions, ${ }^{87,88}$ liposomes, ${ }^{89,90}$ and $\mathrm{PMs},{ }^{91,92}$ have been reported. Ultrasound waves could produce thermal (by absorption of energy) and nonthermal/ mechanical effects by the cavitation, resulting in the destabilization of nanopreparations and resultant drug release. In addition, ultrasound can also increase the permeability of biological barriers, such as cell membrane and blood-brain barrier, through the formation of cavitation bubbles and generated heat, leading to enhanced cellular uptake of the drugs. ${ }^{93}$ Ultrasound-responsive PMs have shown increased drug release upon exposure to ultrasound by the physical/ chemical disruption of the micelles, representing an attractive drug delivery and release strategy. To date, the most 


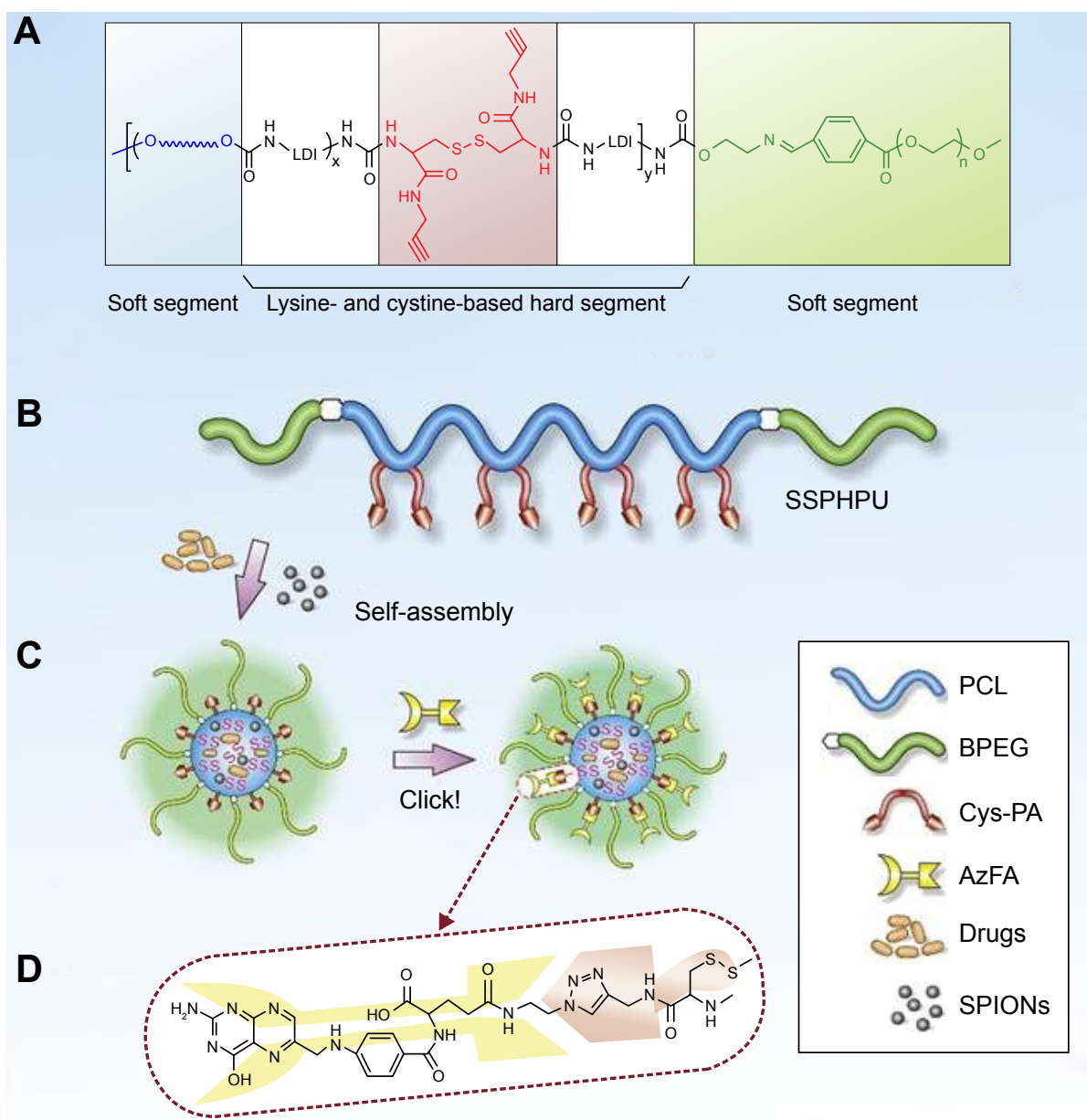

E

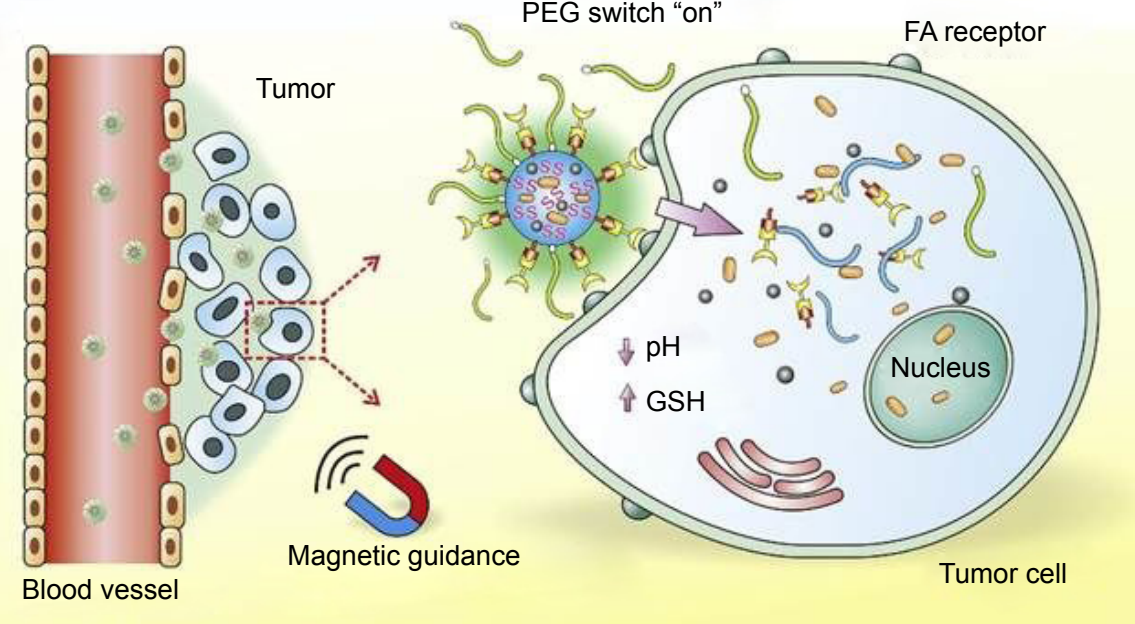

Figure 7 Design of clickable and imageable multiblock polymer micelles. (A) Schematic chemical structure and (B) molecular architecture of clickable MPUs. The waved lines in (A) represent PCL soft segments. (C) Self-assembly of MPU micelles and post-conjugation of FA via click chemistry. (D) Schematic illustration of FA residues on the interface of polymer micelles. (E) Illustration of magnetic-guided and PEG-switched targeting and release properties of MPU nanocarriers.

Note: Reprinted from Wei J, Shuai X, Wang R, et al. Clickable and imageable multiblock polymer micelles with magnetically guided and PEG-switched targeting and release property for precise tumor theranosis. Biomaterials. 2017;145:138-153.80 With permission from Elsevier. Copyright 2017 Elsevier.

Abbreviations: IMPU, multiblock polyurethane; PCL, poly(ع-caprolactone); FA, folate; PEG, poly(ethylene glycol); BPEG, benzoic-imine linkage bearing PEG; Cys-PA, L-cystine-propargylamine; AzFA, azido modified FA; SSPHPU, BPEG-(PCL- Cys-PA)-BPEG; SPIONs, superparamagnetic iron oxide nanoparticles; GSH, glutathione.

extensively studied micelles for ultrasound-triggered drug release are based on Pluronic copolymers that are composed of a ternary copolymer of poly(ethylene oxide) (PEO) and PPO (PEO-PPO-PEO). ${ }^{94,95}$
Recently, Wu et al developed Pluronic P123/F127 PMs encapsulating curcumin that could be permeabilized directly by focused ultrasound, leading to ultrasound-triggered drug release.${ }^{96}$ Compared to free curcumin, curcumin-loaded 
P123/F127 mixed micelles showed longer circulating time and increased cellular uptake. With the assistance of the focused ultrasound, such micellar system exhibited tumortargeted deposition in a time-dependent manner following systemic administration, which was caused by the enhanced permeabilization of tumor regions and increased penetration of PMs in irradiated tumor cells under ultrasound sonoporation. Additionally, the mixed micelles could be regulated by ultrasound irradiation. The in vivo site-specific drug release was demonstrated in dual-tumor models, which showed spatiotemporal release of the entrapped drugs following intratumoral injection, resulting in significant inhibition of the tumor growth by approximately 6.5 -fold more than that of the PMs without ultrasound irradiation. In another interesting work, Liang et al synthesized a metal-supramolecular diblock copolymer (poly(propylene glycol)-PEG) with a mechano-labile bis(terpyridine)-Cu(II) complex linkage in the junction point, which could self-assemble into the spherical PMs in aqueous solution. ${ }^{97}$ They found that the weak $\mathrm{Cu}(\mathrm{II})$-terpyridine dynamic bond in the copolymer chain was able to be cleaved under the high-intensity focused ultrasound, leading to the disruption of the copolymer micelles and the release of the loaded cargo.

\section{Light-responsive PMs}

Among various trigger signals used in biomedical applications, light, including UV, visible, and infrared/NIR light, represents an attractive external stimulus owing to its noninvasive nature, the possibility of remote control, and high spatiotemporal resolution..$^{98-101}$ The use of NIR light is of particular interest due to its deep tissue penetration (up to about $10 \mathrm{~cm}$ in human body) without significant tissue damage, while UV light cannot penetrate deeply into the body as a result of absorption by the skin, blood, and tissues. ${ }^{102}$ In addition, light-induced reactions can be regulated for various applications by adjusting the light parameters like wavelength, intensity, and exposure time. Because of these advantages, a large number of light-responsive NDDSs have been developed to achieve on-demand drug release for cancer treatment. Among them, PMs and liposomes are the most commonly studied ones. The light-responsive PMs are typically obtained by incorporation and/or conjugation of chromophores, such as azobenzene, pyrene, cinnamoyl, spirobenzopyran, or nitrobenzyl groups, in/to the polymeric structure. ${ }^{103-105}$ Upon illumination, the PMs' nanostructure changes and the micelles dissociate, thus releasing the payloads. Due to the ability of reversible UV-visible photoisomerization, azobenzene and its derivatives are one of the most widely studied light-responsive chromophores, which can undergo a trans-to-cis photoisomerization of nitrogen double bond upon the UV irradiation, while this conversion can be reconverted to the trans isomer under the visible light. However, some other chromophores like 2-diazo-1,2-napthoquinone (DNQ) undergo light-responsive irreversible cleavage, converting hydrophobic molecules into hydrophilic ones. ${ }^{106}$ Accordingly, light-responsive PMs are categorized into two types based on the above mechanisms of destabilization, including reversible structural change and irreversible photo-cleavage.

Poelma et al presented one-photon visible light-responsive PMs using the reversible structural change strategy for efficient, on-demand delivery of small molecules. ${ }^{107}$ They developed the donor-acceptor Stenhouse adducts (DASAs) which could undergo a hydrophobic-to-hydrophilic polarity change triggered by visible light between 530 and $570 \mathrm{~nm} .{ }^{108}$ Compared to two-photon processes, molecules with one-photon absorption showed more desirable wavelengths and were more efficient, with the photoisomerization of DASA systems being triggered by low intensities of light $\left(\sim 1 \mathrm{~mW} \mathrm{~cm}^{-2}\right)$. In this study, the DASA building blocks were used as photochromic units in the construction of PMs, allowing for the light-triggered release of small molecules to cells by the structural disassembly of micelles using one-photon visible light. Spiropyran (SP), a well-known photochromic molecule, was exploited by Son et al to initiate the ring-opening multibranching polymerization of glycidol to afford a series of hyperbranched polyglycerol (SP-hb-PG) micelles. ${ }^{109}$ The micelle assembly and disassembly could be induced by an external light source owing to the reversible photoisomerization of hydrophobic SP to hydrophilic merocyanine corresponding to the color change from colorless to pink. The assembly and disassembly of SP-hb-PG micelles were confirmed by a series of experiments, involving transmission electron microscopy (TEM), atomic force microscopy, UV-visible spectroscopy, and dynamic light scattering (DLS).

In addition to the reversible photoisomerization, the lightinduced cleavage of chemical bonds has also been widely used in light-responsive PMs. Saravanakumar et al reported a biocompatible amphiphilic block copolymer $\left({ }^{1} \mathrm{O}_{2}\right.$-PEG- $b$ PCL) formed PMs bearing a singlet oxygen $\left({ }^{1} \mathrm{O}_{2}\right)$-sensitive vinyldithioether cleavable linker at the core-shell junction, which could undergo ${ }^{1} \mathrm{O}_{2}$-mediated photocleavage in the presence of visible light, leading to light-responsive release of ${ }^{1} \mathrm{O}_{2}$ and anticancer drugs for enhanced photodynamic therapy (Figure 8). ${ }^{110}$ The double bond of the vinyldithioether linker can readily react with ${ }^{1} \mathrm{O}_{2}$ to form a dioxetane intermediate, which is unstable and spontaneously cleaved. Unlike other conventional photo-responsive micelles that demand direct 
A

PCL (hydrophobic) PEG (hydrophilic)
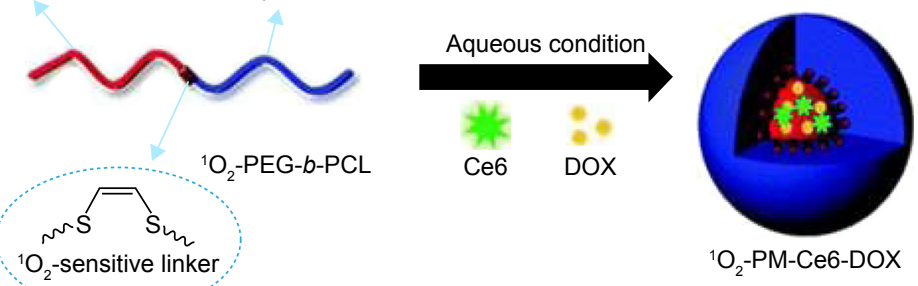

B

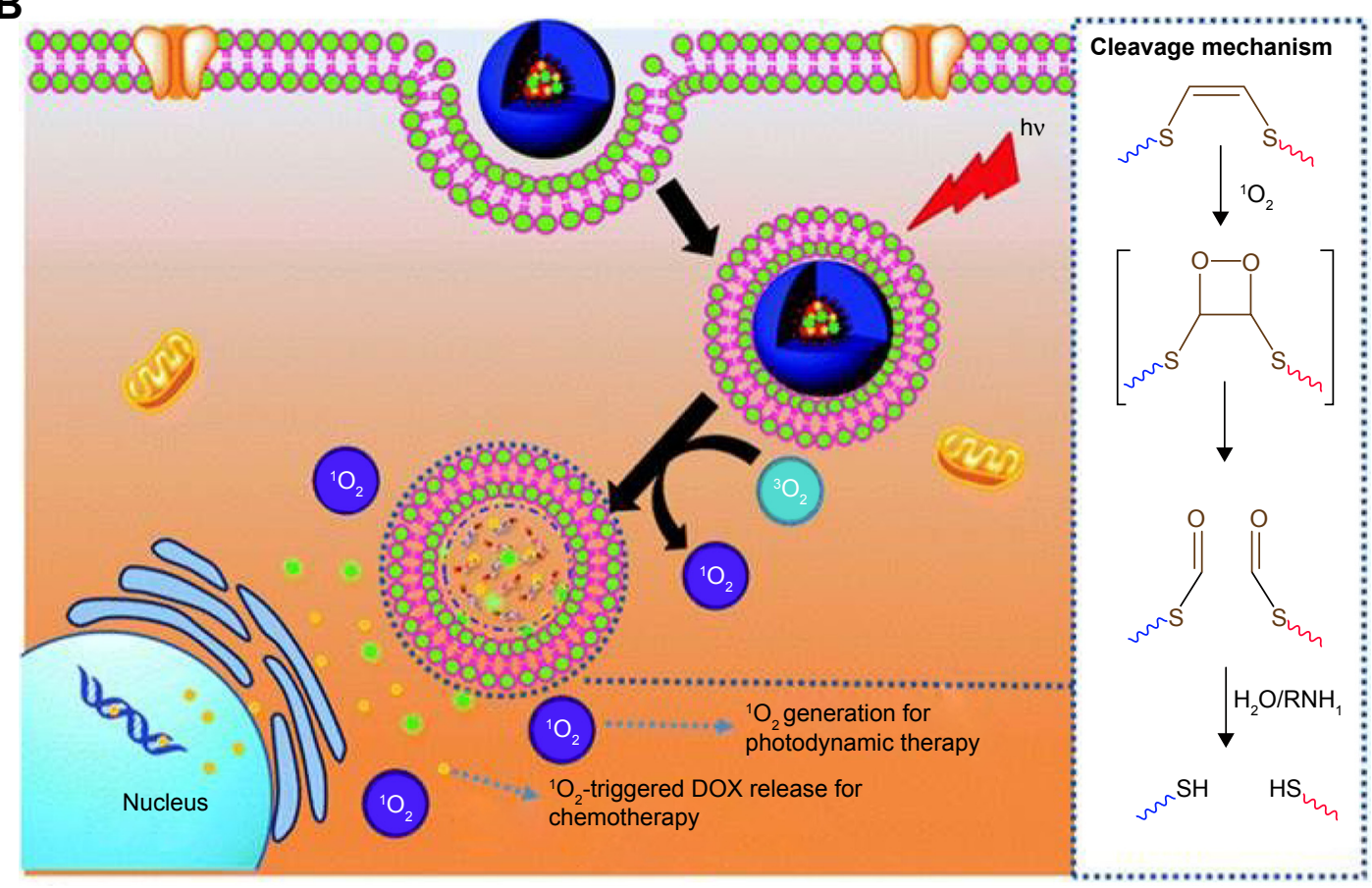

C

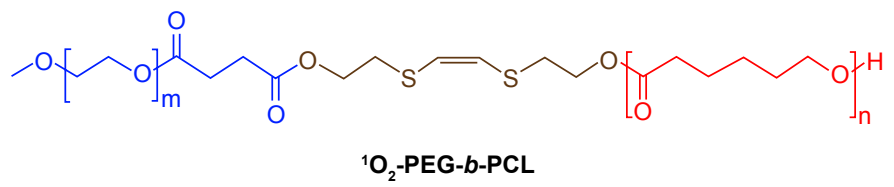

Figure 8 Schematic illustration of $(\mathbf{A})$ the formation of $\mathrm{Ce} 6$ and DOX co-loaded micelles (' $\mathrm{O}_{2}$-PM-Ce6-DOX), (B) cellular uptake and visible light-triggered ' $\mathrm{O}_{2}$-mediated intracellular co-delivery of $\mathrm{Ce} 6$ and DOX for combination chemo- and photodynamic therapy, and (C) chemical structure of the ${ }^{\prime} \mathrm{O}_{2}$-PEG-b-PCL copolymer.

Notes: Reprinted from Saravanakumar G, Lee J, Kim J, Kim WJ. Visible light-induced singlet oxygen-mediated intracellular disassembly of polymeric micelles co-loaded with a photosensitizer and an anticancer drug for enhanced photodynamic therapy. Chem Commun (Camb). 2015;5I (49):9995-9998. ${ }^{110}$ Creative Commons Attribution 3.0 Unported License. Published by the Royal Society of Chemistry.

Abbreviations: Ce6, chlorin e6; DOX, doxorubicin; PM, polymeric micelle; PEG, poly(ethylene glycol); PCL, poly(e-caprolactone).

absorption of UV light, the prepared PMs utilized the ${ }^{1} \mathrm{O}_{2}$ generated by visible light during photodynamic therapy as a trigger to disassemble and release a co-loaded drug in the tumor cell for effective therapy. In another study, Sun et al reported the preparation of an amphiphilic dendritic polymer of PEO-D3DNQ for both UV- and NIR-triggered drug release. ${ }^{111}$ In this work, PEO-D3DNQ was prepared by click conjugation between DNQ-decorated poly(amido amine) dendron D3 (generation 3) and azide-terminated PEO (PEO-N3, molecular weights of 2 and $5 \mathrm{kDa}$ ). As characterized by time-resolved UV-visible spectroscopy, DLS, and TEM, the obtained micelles exhibited both UV and NIR sensitivity in phosphate-buffered saline (PBS). It was found that the characteristic absorption intensity of DNQ progressively decreased and then leveled off within 8 minutes under $\mathrm{UV}_{365 \mathrm{~nm}}$ irradiation, suggesting the completion of the Wolff rearrangement of DNQ, while under $808 \mathrm{~nm}$ NIR irradiation, it took a longer time of 40-60 minutes to complete the Wolff rearrangement of DNQ. After 30 minutes of $808 \mathrm{~nm}$ irradiation, most of the micelles could be disrupted and the apparent drug-release rate showed a nearly eightfold increase, presenting an NIR-triggered drug-release profile.

\section{Multi-responsive PMs}

Multi-stimuli-responsive PMs that are sensitive to two or more stimuli are emerging and have shown great potential 
for cancer-targeted drug delivery and therapy. By combining multiple stimuli sensitivities into one PM system, a precisely controlled drug delivery and release can be achieved, resulting in superior in vitro and/or in vivo anticancer activity. The combined sensitivities to different stimuli can take place either simultaneously or in a sequential way.

\section{Dual $\mathrm{pH} /$ redox-responsive PMs}

Dual pH/redox-responsive PMs have been developed, as both stimuli are naturally present in the cancer microenvironment. Recently, Alex et al prepared a dual redox/pH-responsive PM for co-delivery of DOX and polo-like kinase 1 siRNA. ${ }^{112}$ Cationic structures including histidine and arginine were first grafted to the poly(styrene-altmaleic anhydride) through disulfide linkages, and then short PEG chains were incorporated to impart stealth nature and self-assembly ability. Besides, in order to make them stabile in physiological conditions, drug-loaded PMs were noncovalently coated with BSA. The dual-stimuli-sensitive nature of the PMs significantly increased their drug release at low $\mathrm{pH}$ and $10 \mathrm{mM}$ GSH which mimicked the endosomal $\mathrm{pH}$ and GSH-rich microenvironment of cancer cells. Both in vitro and in vivo results showed synergistic effects of DOX and siRNA in inhibiting tumor cell proliferation.

\section{Dual $\mathrm{pH} /$ thermo-responsive PMs}

Among various dual-stimuli-responsive PMs developed for cancer therapy, $\mathrm{pH}$ and temperature are the most commonly studied combination because of the tumor's acidity and relatively higher local temperature or easily applied external heat sources. Hong et al conjugated PHis to poly(lactic-co-glycolic acid) (PLGA)-PEG-PLGA to form PHis-PLGA-PEG-PLGAPHis copolymers, by which a thermo- and $\mathrm{pH}$-responsive PM was developed. ${ }^{113}$ The temperature- and $\mathrm{pH}$-induced structural changes of the PMs were characterized by alteration in particle size, pyrene fluorescence intensity, and ${ }^{1} \mathrm{H}$ NMR spectra in $\mathrm{D}_{2} \mathrm{O}$. Additionally, the in vitro drug release, cytotoxicity, and intracellular localization further confirmed the temperature- and $\mathrm{pH}$-responsive properties of the copolymer micelles. In another study, Chen et al prepared an optimized, biodegradable, dual temperature/pH-responsive $\mathrm{PM}$ system for enhanced tumor accumulation, in which the hydrophobic segment of dual-responsive copolymer was composed of poly( $N$-(2-hydroxypropyl) methacrylamide dilactate)-co( $N$-(2-hydroxypropyl) methacrylamide-co-histidine). ${ }^{114}$ The poly( $N$-(2-hydroxypropyl) methacrylamide dilactate) had a thermo-reversible LCST suitable for the targeted delivery of a variety of cargos, and histidine was $\mathrm{pH}$ sensitive and widely used in polymeric drug delivery systems.

\section{Dual redox/magnetic-responsive PMs}

The redox-responsive magnetic nanoparticles such as SPIONs have been developed to combine redox-responsive drug release with magnetic targeting and imaging. Recently, Tang et al developed redox-responsive star-shaped magnetic micelles with active-targeted and magnetic-guided functions for cancer therapy. ${ }^{115}$ The magnetic star-shaped micelles were formed by self-assembly of four-arm PEG-PCL copolymers with disulfide bonds as intermediate linkers. DOX and magnetic iron oxide nanoparticles $\left(\mathrm{Fe}_{3} \mathrm{O}_{4}\right)$ were simultaneously encapsulated into the hydrophobic cores of the micelles. The active targeting was rendered by the conjugation of phenylboronic acid ligands that showed high affinity and selectivity to the tumor-overexpressed antigen salic acid. The resultant magnetic PMs enhanced the antitumor efficacy under magnetic field but decreased the adverse effects.

\section{Dual redox/enzyme-responsive PMs}

In a recent study, Zhang et al developed dual enzyme/ redox-responsive PMs with active targeting abilities to achieve rapid intracellular drug release. ${ }^{116}$ In order to overcome the drugs' poor solubility and low stability, camptothecin (CPT) was chemically conjugated to $\mathrm{MPEG}$ via a redox-responsive linker to form polymeric prodrugs. The enzyme-responsive behavior was achieved by introduction of the azo bonds as the linker between the hydrophobic polycaprolactone and hydrophilic PEG segments. The azo bonds can be selectively cleaved by oxidation-reduction enzyme (azoreductase) in the presence of the coenzyme-reduced nicotinamide adenine dinucleotide phosphate. In addition, PBA was conjugated to the end of PEG to realize active tumor targeting and cellular uptake via receptor-mediated endocytosis. In the cytoplasm, CPT could be rapidly released through the dual triggers of enzyme and redox responsiveness and effectively inhibited the tumor proliferation and decreased the side effects.

\section{Triple $\mathrm{pH} /$ thermo/redox-responsive PMs}

In addition to dual-responsive PMs, triple-responsive systems are also attracting increasing interest. Huang et al reported novel triple thermo/pH/reduction-sensitive PMs based on a block copolymer p(poly(ethylene glycol) methyl methacrylate-co-(tert-butyloxycarbonylaminoethyldithioethyl methacrylamide)-co-vinylimidazole) (p(PEG-MEMA-coBoc-Cyst-MMAm-co-VI))-b-PEG (PPBV) for intracellular drug delivery. ${ }^{117}$ The $\mathrm{pH} /$ temperature-responsive behavior of such PMs was observed by both DLS and UV-vis absorbance. When the solution was heated above $21^{\circ} \mathrm{C}$, the size of 
PPBV in PBS (pH 7.4) increased rapidly from 25 to $56 \mathrm{~nm}$. Besides, the size-temperature curves for PPBV in aqueous solution moved toward higher temperatures with decreasing $\mathrm{pH}$ from 7.4 to 6.0. The PTX-loaded micelles showed slow drug release in PBS but rapid release after exposure to a weakly acidic $\mathrm{pH}$ or reductive environment, indicating the $\mathrm{pH}$ - and reduction-sensitive behavior of PPBV. The obtained PMs showed higher cytotoxicity against HepG2 cells due to the increased intracellular PTX concentration, which therefore may serve as promising carriers for cytostatic drugs.

\section{Conclusion}

A large number of preclinical studies on multifunctional PMs have been published, which showed that PMs-based drug delivery systems are promising to be an effective nanomedicine platform for drug delivery and cancer therapy, resulting in enhanced therapeutic index and reduced systemic side effects compared to traditional anticancer drugs. PMs are one of the nanocarriers that can selectively target drug to the tumor though the EPR effect. The small size along with the suitable surface properties such as the hydrophilic PEG corona could prevent the PMs from rapid clearance from the body, allowing for prolonged circulation time and efficient tumor accumulation. Additionally, as most of the anticancer drugs are poorly water-soluble, PMs may be a perfect way for drug solubilization as well as delivery. Moreover, PMs can be further modified with tumor targeting ligands to increase the drug's tumor selectivity and enhance intracellular drug delivery. To date, about nine drug-loaded PMs have been investigated in clinical trials, of which Genexol-PM was approved in 2007 in Korea and marketed in Europe for treating breast cancer and small-cell lung cancer. However, the clinical translation and desired therapeutic effects of PMs are still far from satisfactory. The drug-loaded PMs currently investigated in the clinical trials mostly rely on the passive tumor targeting via the EPR effect; however, their targeting effect in human sometimes is not as pronounced as that obtained in the simple cell and animal models due to the complexity of the tumor and human body. Besides, the premature drug release in the bloodstream and inadequate drug release in the tumor may also account for the inferior therapeutic effects in human patients. Thus, in order to ensure the success of PMs, further modification of micelles is required regarding the drug loading and release, blood circulation, tumor targeting, cellular uptake, and intracellular drug release.

Numerous stimuli-responsive PMs have been developed to deliver anticancer drug in response to a variety of extra/ intracellular stimuli or external triggers, including $\mathrm{pH}$, redox potential, enzymes, temperature, light, ultrasound, and magnetic field, which showed the improved performance in addressing the above-mentioned concerns. Despite the impressive process has been achieved in the design and application of stimuli-responsive PMs for cancer therapy, many challenges and crucial issues still remain to be addressed. First, it is crucial to understand how PMs interact with the biological components. Upon administration, PMs encounter a variety of biological molecules, cells, and tissues/organs. The surface of PMs would be immediately covered with biological molecules such as serum proteins which play an important role in determining the subsequent biodistribution and cellular responses of PMs. Second, off-target effects or insufficient stimuli sensitivity may happen due to the nonspecific distribution of the stimuli, especially internal stimuli. For example, low pH, high concentration of GSH, or upregulation of certain enzymes can also be found in some normal cells or nontumoral tissues. Besides, the heterogeneity of the patients and tumors and the tumor type and stage greatly influence the status of the internal stimuli. Thus, the stimuli sensitivity of PMs should be well studied. Third, the complicated design and difficulties in the scale-up of stimuli-responsive PMs are likely to hamper their clinical translation, which is one major reason why a number of stimuli-responsive PMs have been reported, whereas only a few of them could enter the clinical stage.

Summing up, we reviewed the recent progress of stimuliresponsive PMs for cancer therapy and highlighted the representative examples regarding the stimuli-responsive temporal and spatial drug delivery and release. The potential and progress of stimuli-responsive PMs have been clearly shown in previous studies. In the near future, we believe that the stimuli-responsive PMs would become an important component of clinical cancer therapy.

\section{Acknowledgments}

The authors would like to acknowledge the support from the National Natural Science Foundation of China (grant numbers 81571786, 31771087, 81271687, 31671015) and Shaanxi Science \& Technology Co-ordination \& Innovation Project (2015KTCL03-12). Qing Zhou is grateful to the China Scholarship Council (CSC) for the financial support (CSC number 201603170199).

\section{Disclosure}

The authors report no conflicts of interest in this work.

\section{References}

1. Aly HA. Cancer therapy and vaccination. J Immunol Methods. 2012; 382(1-2):1-23. 
2. Lorusso D, Bria E, Costantini A, Di Maio M, Rosti G, Mancuso A Patients' perception of chemotherapy side effects: expectations, doctorpatient communication and impact on quality of life - an Italian survey. Eur J Cancer Care (Engl). 2017;26(2):e12618.

3. Demaria M, O’Leary MN, Chang J, et al. Cellular senescence promotes adverse effects of chemotherapy and cancer relapse. Cancer Discov. 2017;7(2):165-176.

4. Shi J, Kantoff PW, Wooster R, Farokhzad OC. Cancer nanomedicine: progress, challenges and opportunities. Nat Rev Cancer. 2017;17(1): 20-37.

5. von Roemeling C, Jiang W, Chan CK, Weissman IL, Kim BYS. Breaking down the barriers to precision cancer nanomedicine. Trends Biotechnol. 2017;35(2):159-171.

6. Zhou Q, Zhang L, Wu H. Nanomaterials for cancer therapies Nanotechnol Rev. 2017;6(5):473-496.

7. Gupta AD. A review on recent advancement of cancer therapy using nanoparticles. Biochem Mol Biol Lett. 2017;3(1):104.

8. Yousefpour Marzbali M, Yari Khosroushahi A. Polymeric micelles as mighty nanocarriers for cancer gene therapy: a review. Cancer Chemother Pharmacol. 2017;9(4):637-649.

9. Cho H, Lai TC, Tomoda K, Kwon GS. Polymeric micelles for multidrug delivery in cancer. AAPS PharmSciTech. 2015;16(1):10-20.

10. Varela-Moreira A, Shi Y, Fens MH, Lammers T, Hennink WE, Schiffelers RM. Clinical application of polymeric micelles for the treatment of cancer. Mater Chem Front. 2017;1:1485-1501.

11. Cabral H, Kataoka K. Progress of drug-loaded polymeric micelles into clinical studies. J Control Release. 2014;190:465-476.

12. Li Y, Xiao K, Zhu W, Deng W, Lam KS. Stimuli-responsive crosslinked micelles for on-demand drug delivery against cancers. Adv Drug Deliv Rev. 2014;66:58-73.

13. Nakayama M, Akimoto J, Okano T. Polymeric micelles with stimulitriggering systems for advanced cancer drug targeting. J Drug Target. 2014;22(7):584-599.

14. Jones M, Leroux J. Polymeric micelles - a new generation of colloida drug carriers. Eur J Pharm Biopharm. 1999;48(2):101-111.

15. Sutton D, Nasongkla N, Blanco E, Gao J. Functionalized micellar systems for cancer targeted drug delivery. Pharm Res. 2007;24(6) 1029-1046.

16. Torchilin VP. Structure and design of polymeric surfactant-based drug delivery systems. J Control Release. 2001;73(2-3):137-172.

17. Torchilin VP. Micellar nanocarriers: pharmaceutical perspectives. Pharm Res. 2007;24(1):1-16.

18. Gros L, Ringsdorf H, Schupp H. Polymeric antitumor agents on a molecular and on a cellular level? Angew Chem Int Ed Engl. 1981;20(4): 305-325.

19. Matsumura Y. Poly (amino acid) micelle nanocarriers in preclinical and clinical studies. Adv Drug Deliv Rev. 2008;60(8):899-914.

20. Peer D, Karp JM, Hong S, Farokhzad OC, Margalit R, Langer R. Nanocarriers as an emerging platform for cancer therapy. Nat Nanotechnol. 2007;2(12):751-760.

21. Greish K. Enhanced permeability and retention (EPR) effect for anticancer nanomedicine drug targeting. Cancer Nanotechnology: Methods and Protocols. 2010:25-37.

22. Cabral H, Matsumoto Y, Mizuno K, et al. Accumulation of sub-100 nm polymeric micelles in poorly permeable tumours depends on size. Nat Nanotechnol. 2011;6(12):815-823.

23. Huang K, Ma H, Liu J, et al. Size-dependent localization and penetration of ultrasmall gold nanoparticles in cancer cells, multicellular spheroids, and tumors in vivo. ACS Nano. 2012;6(5):4483-4493.

24. Ma X, Wu Y, Jin S, et al. Gold nanoparticles induce autophagosome accumulation through size-dependent nanoparticle uptake and lysosome impairment. ACS Nano. 2011;5(11):8629-8639.

25. Mahmud A, Xiong XB, Aliabadi HM, Lavasanifar A. Polymeric micelles for drug targeting. J Drug Target. 2007;15(9):553-584.

26. Miller T, Breyer S, van Colen G, et al. Premature drug release of polymeric micelles and its effects on tumor targeting. Int J Pharm. 2013; 445(1-2):117-124
27. Bae Y, Fukushima S, Harada A, Kataoka K. Design of environmentsensitive supramolecular assemblies for intracellular drug delivery: polymeric micelles that are responsive to intracellular $\mathrm{pH}$ change. Angew Chem Int Ed Engl. 2003;42(38):4640-4643.

28. Ko J, Park K, Kim YS, et al. Tumoral acidic extracellular $\mathrm{pH}$ targeting of $\mathrm{pH}$-responsive MPEG-poly (beta-amino ester) block copolymer micelles for cancer therapy. J Control Release. 2007;123(2):109-115.

29. Min KH, Kim JH, Bae SM, et al. Tumoral acidic pH-responsive MPEGpoly (beta-amino ester) polymeric micelles for cancer targeting therapy. J Control Release. 2010;144(2):259-266.

30. Koo H, Lee H, Lee $\mathrm{S}$, et al. In vivo tumor diagnosis and photodynamic therapy via tumoral $\mathrm{pH}$-responsive polymeric micelles. Chem Commun (Camb). 2010;46(31):5668-5670.

31. Kanamala M, Wilson WR, Yang M, Palmer BD, Wu Z. Mechanisms and biomaterials in pH-responsive tumour targeted drug delivery: a review. Biomaterials. 2016;85:152-167.

32. Wu H, Zhu L, Torchilin VP. pH-sensitive poly (histidine)-PEG/ DSPE-PEG co-polymer micelles for cytosolic drug delivery. Biomaterials. 2013;34(4):1213-1222.

33. Hu J, Miura S, Na K, Bae YH. pH-responsive and charge shielded cationic micelle of poly (L-histidine)-block-short branched PEI for acidic cancer treatment. J Control Release. 2013;172(1):69-76.

34. Yang T, Li F, Zhang H, et al. Multifunctional $\mathrm{pH}$-sensitive micelles for tumor-specific uptake and cellular delivery. Polym Chem. 2015; 6(8):1373-1382.

35. Zhou Q, Hou Y, Zhang L, et al. Dual-pH sensitive charge-reversal nanocomplex for tumor-targeted drug delivery with enhanced anticancer activity. Theranostics. 2017;7(7):1806-1819.

36. Zhou Z, Li L, Yang Y, Xu X, Huang Y. Tumor targeting by $\mathrm{pH}$-sensitive, biodegradable, cross-linked N-(2-hydroxypropyl) methacrylamide copolymer micelles. Biomaterials. 2014;35(24):6622-6635.

37. Mintzer MA, Simanek EE. Nonviral vectors for gene delivery. Chem Rev. 2008;109(2):259-302.

38. Anderson DG, Lynn DM, Langer R. Semi-automated synthesis and screening of a large library of degradable cationic polymers for gene delivery. Angew Chem. 2003;115(27):3261-3266.

39. Lee HJ, Pardridge WM. Monoclonal antibody radiopharmaceuticals: cationization, pegylation, radiometal chelation, pharmacokinetics, and tumor imaging. Bioconjug Chem. 2003;14(3):546-553.

40. Ma SF, Nishikawa M, Katsumi H, Yamashita F, Hashida M. Cationic charge-dependent hepatic delivery of amidated serum albumin. J Control Release. 2005;102(3):583-594.

41. Balendiran GK, Dabur R, Fraser D. The role of glutathione in cancer. Cell Biochem Funct. 2004;22(6):343-352.

42. Schafer FQ, Buettner GR. Redox environment of the cell as viewed through the redox state of the glutathione disulfide/glutathione couple. Free Radic Biol Med. 2001;30(11):1191-1212.

43. Kuppusamy P, Li H, Ilangovan G, et al. Noninvasive imaging of tumor redox status and its modification by tissue glutathione levels. Cancer Res. 2002;62(1):307-312.

44. Sun Y, Yan X, Yuan T, et al. Disassemblable micelles based on reduction-degradable amphiphilic graft copolymers for intracellular delivery of doxorubicin. Biomaterials. 2010;31(27):7124-7131.

45. Li J, Huo M, Wang J, et al. Redox-sensitive micelles self-assembled from amphiphilic hyaluronic acid-deoxycholic acid conjugates for targeted intracellular delivery of paclitaxel. Biomaterials. 2012;33(7): 2310-2320.

46. Wang YC, Wang F, Sun TM, Wang J. Redox-responsive nanoparticles from the single disulfide bond-bridged block copolymer as drug carriers for overcoming multidrug resistance in cancer cells. Bioconjug Chem. 2011;22(10):1939-1945

47. Shi C, Guo X, Qu Q, Tang Z, Wang Y, Zhou S. Actively targeted delivery of anticancer drug to tumor cells by redox-responsive starshaped micelles. Biomaterials. 2014;35(30):8711-8722.

48. Wang J, Yang G, Guo X, Tang Z, Zhong Z, Zhou S. Redox-responsive polyanhydride micelles for cancer therapy. Biomaterials. 2014;35(9): 3080-3090. 
49. Hu J, Zhang G, Liu S. Enzyme-responsive polymeric assemblies, nanoparticles and hydrogels. Chem Soc Rev. 2012;41(18):5933-5949.

50. Egeblad M, Werb Z. New functions for the matrix metalloproteinases in cancer progression. Nat Rev Cancer. 2002;2(3):161-174.

51. Kessenbrock K, Plaks V, Werb Z. Matrix metalloproteinases: regulators of the tumor microenvironment. Cell. 2010;141(1):52-67.

52. Park JB, Lee CS, Jang JH, et al. Phospholipase signalling networks in cancer. Nat Rev Cancer. 2012;12(11):782-792.

53. Hu Q, Katti PS, Gu Z. Enzyme-responsive nanomaterials for controlled drug delivery. Nanoscale. 2014;6(21):12273-12286.

54. Choi KY, Yoon HY, Kim JH, et al. Smart nanocarrier based on PEGylated hyaluronic acid for cancer therapy. ACS Nano. 2011;5(11):8591-8599.

55. Jiang T, Mo R, Bellotti A, Zhou J, Gu Z. Gel-liposome-mediated codelivery of anticancer membrane-associated proteins and small-molecule drugs for enhanced therapeutic efficacy. Adv Funct Mater. 2014;24(16): 2295-2304.

56. Xu JH, Gao FP, Li LL, et al. Gelatin-mesoporous silica nanoparticles as matrix metalloproteinases-degradable drug delivery systems in vivo. Microporous Mesoporous Mater. 2013;182:165-172.

57. Zhu L, Wang T, Perche F, Taigind A, Torchilin VP. Enhanced anticancer activity of nanopreparation containing an MMP2-sensitive PEG-drug conjugate and cell-penetrating moiety. Proc Natl Acad Sci U S A. 2013;110(42):17047-17052.

58. Zhu L, Perche F, Wang T, Torchilin VP. Matrix metalloproteinase 2-sensitive multifunctional polymeric micelles for tumor-specific codelivery of siRNA and hydrophobic drugs. Biomaterials. 2014;35(13): 4213-4222.

59. Colotta F, Allavena P, Sica A, Garlanda C, Mantovani A. Cancer-related inflammation, the seventh hallmark of cancer: links to genetic instability. Carcinogenesis. 2009;30(7):1073-1081.

60. Gromkowski SH, Yagi J, Janeway CA Jr. Elevated temperature regulates tumor necrosis factor-mediated immune killing. Eur J Immunol. 1989;19(9):1709-1714.

61. Chen S, Li Y, Guo C, et al. Temperature-responsive magnetite/ PEO-PPO-PEO block copolymer nanoparticles for controlled drug targeting delivery. Langmuir. 2007;23(25):12669-12676.

62. Koppolu B, Bhavsar Z, Wadajkar AS, et al. Temperature-sensitive polymer-coated magnetic nanoparticles as a potential drug delivery system for targeted therapy of thyroid cancer. J Biomed Nanotechnol. 2012;8(6):983-990.

63. Purushotham S, Chang P, Rumpel H, et al. Thermoresponsive coreshell magnetic nanoparticles for combined modalities of cancer therapy. Nanotechnology. 2009;20(30):305101.

64. Sun JT, Yu ZQ, Hong CY, Pan CY. Biocompatible zwitterionic sulfobetaine copolymer-coated mesoporous silica nanoparticles for temperature-responsive drug release. Macromol Rapid Commun. 2012; 33(9):811-818.

65. Kanazawa H, Yamamoto K, Matsushima Y, et al. Temperature-responsive chromatography using poly ( $\mathrm{N}$-isopropylacrylamide)-modified silica. Anal Chem. 1996;68(1):100-105.

66. Ron ES, Bromberg LE. Temperature-responsive gels and thermogelling polymer matrices for protein and peptide delivery. Adv Drug Deliv Rev. 1998;31(3):197-221.

67. Nakayama M, Okano T, Miyazaki T, Kohori F, Sakai K, Yokoyama M. Molecular design of biodegradable polymeric micelles for temperatureresponsive drug release. J Control Release. 2006;115(1):46-56.

68. Wei H, Zhang X, Cheng C, Cheng SX, Zhuo RX. Self-assembled, thermosensitive micelles of a star block copolymer based on PMMA and PNIPAAm for controlled drug delivery. Biomaterials. 2007; 28(1):99-107.

69. Zhang XZ, Wu DQ, Chu CC. Synthesis, characterization and controlled drug release of thermosensitive IPN-PNIPAAm hydrogels. Biomaterials. 2004;25(17):3793-3805.

70. Zhang X, Wu D, Chu CC. Synthesis and characterization of partially biodegradable, temperature and $\mathrm{pH}$ sensitive Dex-MA/PNIPAAm hydrogels. Biomaterials. 2004;25(19):4719-4730.
71. Schmaljohann D, Oswald J, Jørgensen B, Nitschke M, Beyerlein D, Werner C. Thermo-responsive PNiPAAm-g-PEG films for controlled cell detachment. Biomacromolecules. 2003;4(6):1733-1739.

72. Panja S, Dey G, Bharti R, et al. Tailor-made temperature-sensitive micelle for targeted and on-demand release of anticancer drugs. ACS Appl Mater Interfaces. 2016;8(19):12063-12074.

73. Ahn DG, Lee J, Park SY, Kwark YJ, Lee KY. Doxorubicin-loaded alginate-g-poly (N-isopropylacrylamide) micelles for cancer imaging and therapy. ACS Appl Mater Interfaces. 2014;6(24):22069-22077.

74. Sawant RM, Sawant RR, Gultepe E, et al. Nanosized cancer cell-targeted polymeric immunomicelles loaded with superparamagnetic iron oxide nanoparticles. J Nanopart Res. 2009;11(7):1777.

75. Liao C, Sun Q, Liang B, Shen J, Shuai X. Targeting EGFR-overexpressing tumor cells using Cetuximab-immunomicelles loaded with doxorubicin and superparamagnetic iron oxide. Eur J Radiol. 2011;80(3):699-705.

76. Gupta AK, Naregalkar RR, Vaidya VD, Gupta M. Recent advances on surface engineering of magnetic iron oxide nanoparticles and their biomedical applications. Nanomedicine (Lond). 2007;2(1):23-39.

77. Gupta AK, Gupta M. Synthesis and surface engineering of iron oxide nanoparticles for biomedical applications. Biomaterials. 2005;26(18): 3995-4021.

78. Shinkai M. Functional magnetic particles for medical application. J Biosci Bioeng. 2002;94(6):606-613.

79. Boyer C, Whittaker MR, Bulmus V, Liu J, Davis TP. The design and utility of polymer-stabilized iron-oxide nanoparticles for nanomedicine applications. NPG Asia Mater. 2010;2(1):23-30.

80. Wei J, Shuai X, Wang R, et al. Clickable and imageable multiblock polymer micelles with magnetically guided and PEG-switched targeting and release property for precise tumor theranosis. Biomaterials. 2017; 145:138-153.

81. Li H, Yan K, Shang Y, et al. Folate-bovine serum albumin functionalized polymeric micelles loaded with superparamagnetic iron oxide nanoparticles for tumor targeting and magnetic resonance imaging. Acta Biomater. 2015;15:117-126.

82. Lee SY, Yang CY, Peng CL, et al. A theranostic micelleplex codelivering SN-38 and VEGF siRNA for colorectal cancer therapy. Biomaterials. 2016;86:92-105.

83. Mitragotri S. Healing sound: the use of ultrasound in drug delivery and other therapeutic applications. Nat Rev Drug Discov. 2005;4(3): 255-260.

84. Chen Y, Chen H, Sun Y, et al. Multifunctional mesoporous composite nanocapsules for highly efficient MRI-guided high-intensity focused ultrasound cancer surgery. Angew Chem Int Ed Engl. 2011;50(52): $12505-12509$.

85. Wang X, Chen H, Chen Y, et al. Perfluorohexane-encapsulated mesoporous silica nanocapsules as enhancement agents for highly efficient High Intensity Focused Ultrasound (HIFU). Adv Mater. 2012; 24(6):785-791

86. Yang P, Li D, Jin S, et al. Stimuli-responsive biodegradable poly (methacrylic acid) based nanocapsules for ultrasound traced and triggered drug delivery system. Biomaterials. 2014;35(6):2079-2088.

87. Rapoport NY, Kennedy AM, Shea JE, Scaife CL, Nam KH. Controlled and targeted tumor chemotherapy by ultrasound-activated nanoemulsions/ microbubbles. J Control Release. 2009;138(3):268-276.

88. Rapoport N, Nam KH, Gupta R, et al. Ultrasound-mediated tumor imaging and nanotherapy using drug loaded, block copolymer stabilized perfluorocarbon nanoemulsions. J Control Release. 2011;153(1):4-15.

89. Huang SL, MacDonald RC. Acoustically active liposomes for drug encapsulation and ultrasound-triggered release. Biochim Biophys Acta. 2004;1665(1-2):134-141.

90. Schroeder A, Honen R, Turjeman K, Gabizon A, Kost J, Barenholz Y. Ultrasound triggered release of cisplatin from liposomes in murine tumors. J Control Release. 2009;137(1):63-68.

91. Zhang H, Xia H, Wang J, Li Y. High intensity focused ultrasoundresponsive release behavior of PLA-b-PEG copolymer micelles. J Control Release. 2009;139(1):31-39. 
92. Mohan P, Rapoport N. Doxorubicin as a molecular nanotheranostic agent: effect of doxorubicin encapsulation in micelles or nanoemulsions on the ultrasound-mediated intracellular delivery and nuclear trafficking. Mol Pharm. 2010;7(6):1959-1973.

93. Unger EC, McCreery TP, Sweitzer RH. Ultrasound enhances gene expression of liposomal transfection. Invest Radiol. 1997;32(12): 723-727.

94. Marin A, Sun H, Husseini GA, Pitt WG, Christensen DA, Rapoport NY. Drug delivery in pluronic micelles: effect of high-frequency ultrasound on drug release from micelles and intracellular uptake. $J$ Control Release. 2002;84(1-2):39-47.

95. Husseini GA, Christensen DA, Rapoport NY, Pitt WG. Ultrasonic release of doxorubicin from Pluronic P105 micelles stabilized with an interpenetrating network of N, N-diethylacrylamide. J Control Release. 2002;83(2):303-305.

96. Wu P, Jia Y, Qu F, et al. Ultrasound-responsive polymeric micelles for sonoporation-assisted site-specific therapeutic action. ACS Appl Mater Interfaces. 2017;9(31):25706-25716.

97. Liang B, Tong R, Wang Z, Guo S, Xia H. High intensity focused ultrasound responsive metallo-supramolecular block copolymer micelles. Langmuir. 2014;30(31):9524-9532.

98. Shanmugam V, Selvakumar S, Yeh CS. Near-infrared light-responsive nanomaterials in cancer therapeutics. Chem Soc Rev. 2014;43(17): 6254-6287.

99. Angelatos AS, Radt B, Caruso F. Light-responsive polyelectrolyte/ gold nanoparticle microcapsules. J Phys Chem B. 2005;109(7): 3071-3076.

100. Yang X, Liu X, Liu Z, Pu F, Ren J, Qu X. Near-infrared light-triggered, targeted drug delivery to cancer cells by aptamer gated nanovehicles. Adv Mater. 2012;24(21):2890-2895.

101. Zhang Z, Wang J, Chen C. Near-infrared light-mediated nanoplatforms for cancer thermo-chemotherapy and optical imaging. Adv Mater. 2013; 25(28):3869-3880.

102. Jochum FD, Theato P. Temperature- and light-responsive smart polymer materials. Chem Soc Rev. 2013;42(17):7468-7483.

103. Zhao Y. Rational design of light-controllable polymer micelles. Chem Rec. 2007;7(5):286-294.

104. Schumers JM, Fustin CA, Gohy JF. Light-responsive block copolymers. Macromol Rapid Commun. 2010;31(18):1588-1607.

105. Zhao YL, Stoddart JF. Azobenzene-based light-responsive hydrogel system. Langmuir. 2009;25(15):8442-8446.

106. Cui J, Del Campo A. Photo-responsive polymers: properties, synthesis and applications. In: de Armas MRA, Aguilar MR, Román JS, editors. Smart Polymers and Their Applications. Cambridge: Elsevier; 2014: 93-133.

107. Poelma SO, Oh SS, Helmy S, et al. Controlled drug release to cancer cells from modular one-photon visible light-responsive micellar system. Chem Commun. 2016;52(69):10525-10528.

108. Helmy S, Leibfarth FA, Oh S, Poelma JE, Hawker CJ, Read de Alaniz J. Photoswitching using visible light: a new class of organic photochromic molecules. J Am Chem Soc. 2014;136(23):8169-8172.

109. Son S, Shin E, Kim BS. Light-responsive micelles of spiropyran initiated hyperbranched polyglycerol for smart drug delivery. Biomacromolecules. 2014;15(2):628-634.

110. Saravanakumar G, Lee J, Kim J, Kim WJ. Visible light-induced singlet oxygen-mediated intracellular disassembly of polymeric micelles co-loaded with a photosensitizer and an anticancer drug for enhanced photodynamic therapy. Chem Commun (Camb). 2015; 51(49):9995-9998.

111. Sun L, Zhu B, Su Y, Dong CM. Light-responsive linear-dendritic amphiphiles and their nanomedicines for NIR-triggered drug release. Polym Chem. 2014;5(5):1605-1613.

112. Alex MA, Nehate C, Veeranarayanan S, Kumar DS, Kulshreshtha R, Koul V. Self assembled dual responsive micelles stabilized with protein for co-delivery of drug and siRNA in cancer therapy. Biomaterials. 2017; 133:94-106.
113. Hong W, Chen D, Jia L, et al. Thermo- and pH-responsive copolymers based on PLGA-PEG-PLGA and poly (L-histidine): synthesis and in vitro characterization of copolymer micelles. Acta Biomater. 2014;10(3):1259-1271

114. Chen YC, Liao LC, Lu PL, et al. The accumulation of dual $\mathrm{pH}$ and temperature responsive micelles in tumors. Biomaterials. 2012;33(18): 4576-4588.

115. Tang Z, Zhang L, Wang Y, Li D, Zhong Z, Zhou S. Redoxresponsive star-shaped magnetic micelles with active-targeted and magnetic-guided functions for cancer therapy. Acta Biomater. 2016; 42:232-246.

116. Zhang L, Wang Y, Zhang X, Wei X, Xiong X, Zhou S. Enzyme and redox dual-triggered intracellular release from actively targeted polymeric micelles. ACS Appl Mater Interfaces. 2017;9(4):3388-3399.

117. Huang X, Jiang X, Yang Q, et al. Triple-stimuli ( $\mathrm{pH} /$ thermo/reduction) sensitive copolymers for intracellular drug delivery. J Mater Chem B. 2013;1(13):1860-1868.

118. Valle JW, Armstrong A, Newman C, et al. A phase 2 study of SP1049C, doxorubicin in P-glycoprotein-targeting pluronics, in patients with advanced adenocarcinoma of the esophagus and gastroesophageal junction. Invest New Drugs. 2011;29(5):1029-1037.

119. Danson S, Ferry D, Alakhov V, et al. Phase I dose escalation and pharmacokinetic study of pluronic polymer-bound doxorubicin (SP1049C) in patients with advanced cancer. Br J Cancer. 2004;90(11):2085-2091.

120. Armstrong A, Brewer J, Newman C, et al. SP1049C as first-line therapy in advanced (inoperable or metastatic) adenocarcinoma of the oesophagus: a phase II window study. J Clin Oncol. 2006; 24(18 Suppl):4080.

121. Matsumura Y, Kataoka K. Preclinical and clinical studies of anticancer agent-incorporating polymer micelles. Cancer Sci. 2009;100(4): 572-579.

122. Nakanishi T, Fukushima S, Okamoto K, et al. Development of the polymer micelle carrier system for doxorubicin. J Control Release. 2001; 74(1-3):295-302.

123. Kim KS, Park W, Hu J, Bae YH, Na K. A cancer-recognizable MRI contrast agents using $\mathrm{pH}$-responsive polymeric micelle. Biomaterials. 2014;35(1):337-343.

124. Yu P, Yu H, Guo C, et al. Reversal of doxorubicin resistance in breast cancer by mitochondria-targeted $\mathrm{pH}$-responsive micelles. Acta Biomater. 2015;14:115-124.

125. Yu J, Deng H, Xie F, Chen W, Zhu B, Xu Q. The potential of pHresponsive PEG-hyperbranched polyacylhydrazone micelles for cancer therapy. Biomaterials. 2014;35(9):3132-3144.

126. Tan Y, Zhu Y, Zhao Y, et al. Mitochondrial alkaline $\mathrm{pH}$-responsive drug release mediated by Celastrol loaded glycolipid-like micelles for cancer therapy. Biomaterials. 2018;154:169-181.

127. Wen H, Li Y. Redox sensitive nanoparticles with disulfide bond linked sheddable shell for intracellular drug delivery. Med Chem. 2014;4(11):748-755

128. Yin T, Wang L, Yin L, Zhou J, Huo M. Co-delivery of hydrophobic paclitaxel and hydrophilic AURKA specific siRNA by redox-sensitive micelles for effective treatment of breast cancer. Biomaterials. 2015; 61:10-25.

129. Ma YC, Wang JX, Tao W, et al. Redox-responsive polyphosphoesterbased micellar nanomedicines for overriding chemoresistance in breast cancer cells. ACS Appl Mater Interfaces. 2015;7(47):26315-26325.

130. Chen WH, Luo GF, Lei Q, et al. MMP-2 responsive polymeric micelles for cancer-targeted intracellular drug delivery. Chem Commun (Camb). 2015;51(3):465-468.

131. Kalafatovic D, Nobis M, Javid N, et al. MMP-9 triggered micelleto-fibre transitions for slow release of doxorubicin. Biomater Sci. 2015;3(2):246-249.

132. Sahu A, Bora U, Kasoju N, Goswami P. Synthesis of novel biodegradable and self-assembling methoxy poly(ethylene glycol)-palmitate nanocarrier for curcumin delivery to cancer cells. Acta Biomater. 2008; 4(6):1752-1761. 
133. Cheng C, Wei H, Shi BX, et al. Biotinylated thermoresponsive micelle self-assembled from double-hydrophilic block copolymer for drug delivery and tumor target. Biomaterials. 2008;29(4):497-505.

134. Emamzadeh M, Desmaële D, Couvreur P, Pasparakis G. Dual controlled delivery of squalenoyl-gemcitabine and paclitaxel using thermo-responsive polymeric micelles for pancreatic cancer. J Control Release. 2017;259:e90-e91.

135. Hu Y, Darcos V, Monge S, Li S, Zhou Y, Su F. Thermo-responsive release of curcumin from micelles prepared by self-assembly of amphiphilic P(NIPAAm-co-DMAAm)-b-PLLA-b-P(NIPAAm-coDMAAm) triblock copolymers. Int J Pharm. 2014;476(1-2):31-40.

136. Hu Y, Darcos V, Monge S, Li S. Thermo-responsive drug release from self-assembled micelles of brush-like PLA/PEG analogues block copolymers. Int J Pharm. 2015;491(1-2):152-161.

137. Huang C, Tang Z, Zhou Y, et al. Magnetic micelles as a potential platform for dual targeted drug delivery in cancer therapy. Int J Pharm. 2012;429(1-2):113-122.

138. Yang X, Chen Y, Yuan R, et al. Folate-encoded and $\mathrm{Fe}_{3} \mathrm{O}_{4}$-loaded polymeric micelles for dual targeting of cancer cells. Polymer. 2008; 49(16):3477-3485.

139. Glover AL, Bennett JB, Pritchett JS, et al. Magnetic heating of iron oxide nanoparticles and magnetic micelles for cancer therapy. IEEE Trans Magn. 2013;49(1):231-235.

140. Chen CJ, Liu GY, Shi YT, et al. Biocompatible micelles based on comb-like PEG derivates: formation, characterization, and photoresponsiveness. Macromol Rapid Commun. 2011;32(14):1077-1081.

141. Ji W, Li N, Chen D, et al. Coumarin-containing photo-responsive nanocomposites for NIR light-triggered controlled drug release via a two-photon process. J Mater Chem B. 2013;1(43):5942-5949.
142. Pruitt JD, Pitt WG. Sequestration and ultrasound-induced release of doxorubicin from stabilized Pluronic P105 micelles. Drug Deliv. 2002;9(4):253-258.

143. Husseini GA, Rapoport NY, Christensen DA, Pruitt JD, Pitt WG. Kinetics of ultrasonic release of doxorubicin from pluronic P105 micelles. Colloids Surf B Biointerfaces. 2002;24(3-4):253-264.

144. Husseini GA, Abdel-Jabbar NM, Mjalli FS, Pitt WG. Modeling and sensitivity analysis of acoustic release of doxorubicin from unstabilized pluronic P105 using an artificial neural network model. Technol Cancer Res Treat. 2007;6(1):49-56.

145. Chen W, Zhong P, Meng F, et al. Redox and pH-responsive degradable micelles for dually activated intracellular anticancer drug release. J Control Release. 2013;169(3):171-179.

146. Yu H, Cui Z, Yu P, et al. pH- and NIR light-responsive micelles with hyperthermia-triggered tumor penetration and cytoplasm drug release to reverse doxorubicin resistance in breast cancer. Adv Funct Mater. 2015;25(17):2489-2500.

147. Xuan J, Han D, Xia H, Zhao Y. Dual-stimuli-responsive micelle of an $\mathrm{ABC}$ triblock copolymer bearing a redox-cleavable unit and a photocleavable unit at two block junctions. Langmuir. 2014;30(1):410-417.

148. Zou H, Yuan W. Temperature- and redox-responsive magnetic complex micelles for controlled drug release. J Mater Chem B. 2015;3(2): 260-269.

149. Lee SY, Lee H, In I, Park SY. pH/redox/photo responsive polymeric micelle via boronate ester and disulfide bonds with spiropyran-based photochromic polymer for cell imaging and anticancer drug delivery. Eur Polym J. 2014;57:1-10.
International Journal of Nanomedicine

\section{Publish your work in this journal}

The International Journal of Nanomedicine is an international, peerreviewed journal focusing on the application of nanotechnology in diagnostics, therapeutics, and drug delivery systems throughout the biomedical field. This journal is indexed on PubMed Central, MedLine, CAS, SciSearch ${ }^{\circledR}$, Current Contents ${ }^{\circledR} /$ Clinical Medicine,

\section{Dovepress}

Journal Citation Reports/Science Edition, EMBase, Scopus and the Elsevier Bibliographic databases. The manuscript management system is completely online and includes a very quick and fair peer-review system, which is all easy to use. Visit http://www.dovepress.com/ testimonials.php to read real quotes from published authors. 